\title{
BAND SUMS OF LINKS WHICH YIELD COMPOSITE LINKS. THE CABLING CONJECTURE FOR STRONGLY INVERTIBLE KNOTS
}

\author{
MARIO EUDAVE-MUÑOZ
}

\begin{abstract}
We consider composite links obtained by bandings of another link. It is shown that if a banding of a split link yields a composite knot then there is a decomposing sphere crossing the band in one arc, unless there is such a sphere disjoint from the band. We also prove that if a banding of the trivial knot yields a composite knot or link then there is a decomposing sphere crossing the band in one arc. The last theorem implies, via double branched covers, that the only way we can get a reducible manifold by surgery on a strongly invertible knot is when the knot is cabled and the surgery is via the slope of the cabling annulus.
\end{abstract}

\section{INTRODUCTION}

We consider here the problem of when a banding of a link $k$ yields a composite link. There is a trivial situation in which a composite link is produced. This is when one of the summands of $k$ is not perturbed by the band, i.e., there is a decomposing sphere disjoint from the band. There are other cases which are not trivial, for example the square knot is a band sum of trivial knots (Figure 1).

Definitions. A link $k$ is split (or splittable) if there is a sphere $Q$ disjoint from $k$ which separates its components. Such a sphere is called a splitting sphere. Note that $k$ is split if and only if $S^{3}-k$ is reducible.

A link $k$ is composite if there is a sphere $P$ intersecting $k$ transversally in two points, such that neither of the 3-balls bounded by $P$ intersects $k$ in a single unknotted spanning arc. Such a sphere is called a decomposing sphere for $k$. A link is prime if it is neither composite, nor split, nor trivial.

Let $P$ be a decomposing sphere for a composite link $k$. Take a tubular neighborhood $\eta\left(k^{\prime}\right)$ of the component $k^{\prime}$ of $k$ which meets $P . \partial \eta\left(k^{\prime}\right)-P$ consists of two annuli. Attach one of these annuli to $P-$ int $\eta\left(k^{\prime}\right)$, to get a torus $T$. Call $T$ a swallow-follow torus for $k$. There is a disk $D$ such that $D \cap T=\partial D$ and $k$ intersects $D$ transversally in one point. By doing 2surgery on $T$ with $D$ we recover the decomposing sphere. Note that in this construction we get two swallow-follow torus.

Received by the editors May 15, 1989.

1980 Mathematics Subject Classification (1985 Revision). Primary 57M25; Secondary 57N10.

Key words and phrases. Band sum, banding, composite knot and link, decomposing sphere, split link, sutured manifold, surgery on knots.

Research supported in part by a National Science Foundation Grant. 


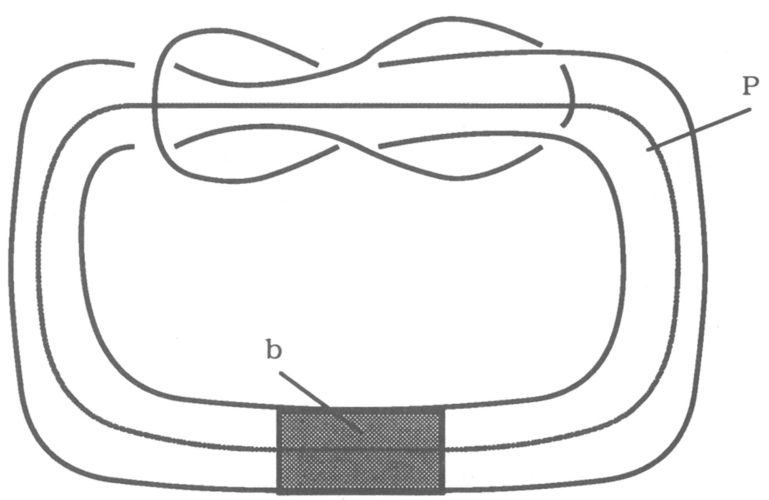

FIGURE 1

Let $k$ be a link in $S^{3}$. Let $b: I \times I \rightarrow S^{3}$ be an imbedding such that $b(I \times I) \cap k=b(I \times \partial I)$. Let $k_{b}=\{k-b(I \times \partial I)\} \cup b(\partial I \times I) . k_{b}$ is another link called a banding of $k$. If $k=k_{0} \cup k_{1}$ is a split link, where $k_{0}, k_{1}$ are knots, and $b(I \times\{i\}) \cap k=b(I \times I) \cap k_{j}$, for $i=0,1$, then $k_{b}$ is called a band sum of $k_{0}$ and $k_{1}$.

A band is trivial if there is a sphere in $S^{3}-k$ which intersects the band in one arc parallel to $b(I \times\{1 / 2\})$. So if $k_{b}$ is a band sum of $k_{0}$ and $k_{1}, b$ is trivial if and only if it is a connected sum of $k_{0}$ and $k_{1}$.

A Seifert surface for a link $k$ is a compact, oriented surface none of whose components are closed and whose boundary is the link. Define $\chi(k)$ to be the maximal Euler characteristic of all Seifert surfaces for $k$.

Let $k$ be a link, and $b$ a band such that $k_{b}$ is composite. If $P$ is a decomposing sphere for $k_{b}$, then it is possible to isotope $P$ so that it meets $k_{b}$ in two points not in $b(\partial I \times I)$, and intersects the band in a collection of arcs parallel to $b(\{1 / 2\} \times I)$. For example, in Figure 1 there is a decomposing sphere crossing the band in one arc. Our main results are the following.

Theorem 1. Let $k$ be a link and $b$ a band such that $S^{3}-(k \cup b(I \times I))$ is irreducible. If $k_{b}$ is composite then either

(a) there is a decomposing sphere for $k_{b}$ disjoint from the band; or

(b) there is a decomposing sphere for $k_{b}$ which crosses the band exactly in one arc parallel to $b(\{1 / 2\} \times I)$; or

(c) there is a swallow-follow torus for $k_{b}$ disjoint from the band; or

(d) $S^{3}-k$ is irreducible and there is a Seifert surface $S$ for $k$ with $\chi(S)=$ $\chi(k)$, and such that the band intersects $S$ transversally always in the same direction.

We prove Theorem 1 in $\S \S 1-5$, using sutured manifold theory and a lengthy combinatorial argument.

Theorem 2. Let $k=k_{0} \cup k_{1}$ be a split link, for knots $k_{0}, k_{1}$, and let $k_{b}$ be a band sum of $k_{0}$ and $k_{1}$. If $k_{b}$ is composite then either there is a decomposing sphere disjoint from the band, or there is a decomposing sphere crossing the band in one arc parallel to $b(\{1 / 2\} \times I)$.

Apply Theorem 1 to the more special case of Theorem 2. Note that under the hypothesis of Theorem 2, case (d) of Theorem 1 cannot happen. We use 
the combinatorics of $[\mathrm{BE}]$ to prove that if (c) happens then this is because we are in the trivial case (a). This is shown in $\S 6$.

Theorem 3. Let $k$ be the trivial knot, and $k_{b}$ a banding of $k$. If $k_{b}$ is composite then there is a decomposing sphere which crosses the band in one arc parallel to $b(\{1 / 2\} \times I)$.

Apply Theorem 1 to the case when $k$ is the trivial knot. Note that cases (a) and (c) of Theorem 1 cannot happen. Making a combinatorial argument we prove that if (d) happens then (b) also happens. This is shown in $\S 7$.

Corollary 1. Suppose $k=k_{0} \cup k_{1}$ is a split link, and $k_{b}$ is a band sum of $k_{0}$ and $k_{1}$. Suppose $k_{b}$ is a composite knot and that there is no decomposing sphere disjoint from the band. Then $k_{b}$ has two prime summands, and one of them is a two-bridge knot.

It is proved in [BE] that if a band sum of trivial knots yields a composite knot, then one of the summands is a two-bridge knot.

Corollary 2. Suppose $k$ is the trivial knot and $k_{b}$ is a banding of $k$. If $k_{b}$ is a composite knot or link, then it has two prime summands, one of them is a two-bridge knot or link.

S. A. Bleiler $\left[\mathrm{B}_{2}\right]$ has proved that if $k$ is the trivial knot and $k_{b}$ is composite, then $k_{b}$ has a two-bridge summand.

Theorems 2 and 3 tell us that most of the knots and links obtained by bandings of the trivial knot or a split link are in fact prime, unless there is a summand which is not perturbed by the band. For example, suppose a banding of the trivial knot or a band sum of a split link yields a composite knot or link, and there is no decomposing sphere disjoint from the band. Now do some twists to the band to get a new knot or link; then this new knot or link is in fact prime. This observation is made more precise in Corollary 3 , stated in $\S 7$.

Corollaries 1, 2, 3 are proved via Theorems 2, 3, and tangle theory [L]. Corollary 1 is proved in $\S 6$, and Corollaries 2,3 in $\S 7$. Theorem 3 has an interesting consequence on surgery of knots, specifically we have

Theorem 4. Suppose $k$ is a nontrivial strongly invertible knot in $S^{3}$. If some surgery on $k$ yields a reducible manifold then $k$ is cabled and the surgery is via the slope of the cabling annulus.

This is proved using Theorem 3 , the fact that the manifolds obtained by surgery in a strongly invertible knot are double branched covers of $S^{3}$ [M], and the fact that a link is prime if and only if its double branched cover is irreducible [KT]. This is shown in $\S 8$.

Throughout the paper we assume familiarity with the theory and the notation of sutured manifolds as presented in [ $\left.\mathrm{Sc}_{3}\right]$. The paper should be interpreted as being in the P.L. category. We refer to [R] or [H] for standard facts in knot theory and 3-manifolds.

I would like to express my sincerest gratitude to Professor Martin Scharlemann for his supervision of this work which formed part of my Ph.D. thesis at the University of California at Santa Barbara. I am thankful to the referee for his suggestions. 


\section{Preliminary arguments}

1.1. Let $\beta$ be the planar "eyeglass" 1-complex consisting of two circles $\beta_{0}$ and $\beta_{1}$ and an arc $\beta_{\alpha}$ joining them. Regard $\beta$ as a complex in $\mathbb{R}^{2} \subset \mathbb{R}^{3}$, and let $U$ and $W$ be regular neighborhoods of $\beta$ in $\mathbb{R}^{2}$ and $\mathbb{R}^{3}$ respectively, so that $U$ is a properly embedded planar surface in the genus two handlebody $W$. $\partial U \subset \partial W$ has three components: two of them, denoted by $c_{0}$ and $c_{1}$, are parallel in $U$ to $\beta_{0}$ and $\beta_{1}$ respectively. Denote the third by $c_{\alpha}$. (See Figure 2.)

1.2. Let $k$ be a link in $S^{3}$ and $k_{b}$ be a banding of $k$. Let $M=S^{3}-\operatorname{int} \eta_{k}$. Embed $\beta$ in $M$ in such a way that $\beta_{\alpha}$ runs parallel to $b(\{1 / 2\} \times I)$, and that $\beta_{0}$ and $\beta_{1}$ are parallel to meridian of $k$; we have that $\beta \cap \partial M=\varnothing$. Consider $W$ as a regular neighborhood of $\beta$ in $M$. Consider $M$ as a sutured manifold, all of whose boundary is in $R_{+}$or $R_{-}$, denote it by $(M, \gamma, \beta)$. Suppose $S^{3}-(k \cup b[I \times I])$ is irreducible; this implies that $(M, \gamma, \beta)$ is $\beta$-irreducible and $\partial M$ is $\beta$-incompressible, so $(M, \gamma, \beta)$ is $\beta$-taut. $(M, \gamma)$ may not be $\varnothing$-taut, i.e. may be reducible. ( $\varnothing$ denotes the empty set, to be $\varnothing$-taut means to be taut in the Thurston norm.) With this hypothesis we restate Theorem 1 as follows.

Theorem 1. Let $k$ be $a$ link and $b$ a band such that $k_{b}$ is a composite link. Then either

(a) there is a decomposing sphere for $k_{b}$ disjoint from the band; or

(b) there is a decomposing sphere which crosses the band exactly in one arc parallel to $b(\{1 / 2\} \times I)$; or

(c) there is a swallow-follow torus for $k_{b}$ disjoint from the band; or

(d) $(M, \gamma)$ is $\varnothing$-taut, and there is a $\beta$-taut Seifert surface for $k$ which is also $\varnothing$-taut; or

(e) $(M, \gamma)=\left(S^{1} \times D^{2}, \varnothing\right)$ (i.e. $k$ is the trivial knot), and $k$ bounds a $\beta$-taut disk.

Note that if (a) happens then (c) also happens, but not vice versa. We include both cases because they appear in different ways. (a) is just a trivial case.

It is not difficult to see that (d) and (e) of the above theorem imply (d) of Theorem 1 in the introduction, i.e. if a Seifert surface for $k$ is taut in the Thurston norm then $\chi(S)=\chi(k)$ (for a proof see [ST $2,1.2]$ ).

The proof of this theorem will be as follows: First we take a $\beta$-taut Seifert surface $S$ for $k$, and a decomposing sphere $Q$ for $k_{b}$, which will be considered as a parameterizing surface; then construct a sutured manifold hierarchy,

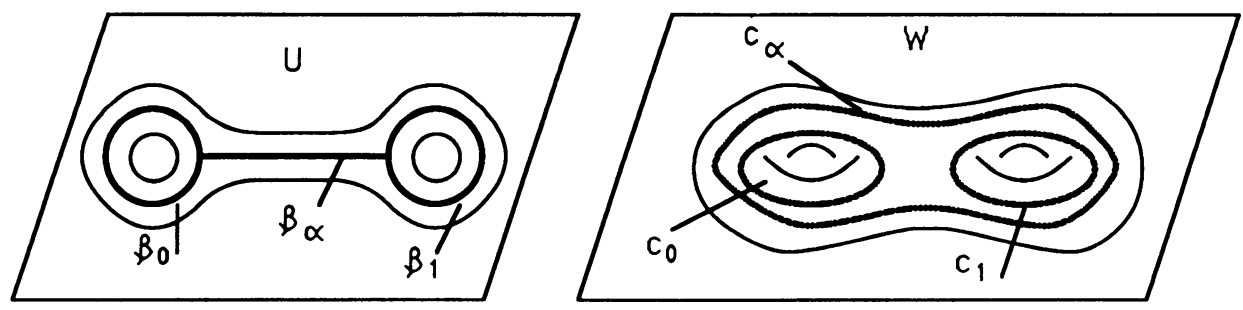

FiguRE 2 
starting with $S$ and respecting $Q$. If the final step in the hierarchy is $\varnothing$-taut, we use [ $\left[\mathrm{Sc}_{4}, 2.7\right.$ and $\left.\mathrm{Sc}_{3}, 3.3\right]$ to get $(\mathrm{d})$ or $(\mathrm{e})$. This is shown in $\S 1$. If the final step in the hierarchy is not $\varnothing$-taut, then we do a lengthy combinatorial argument to see how this final step of the hierarchy may look; we rely heavily on 1.15 and 2.3. This is made in $\S \S 2-4$. In $\S 5$ we use the information gathered to conclude (b) or (c).

1.3. Let $P$ be a decomposing sphere for $k_{b}$. Suppose there is no decomposing sphere disjoint from the band, otherwise we are done. Suppose $P$ intersects the band in a nonempty collection of arcs parallel to $b(\{1 / 2\} \times I)$; suppose this number of arcs taken over all decomposing spheres is minimal. If the band is trivial, then obviously there is a decomposing sphere disjoint from the band, so suppose the band is not trivial. Note that if there is a sphere meeting $\beta$ in one point then the band is trivial.

Assume that the arcs of intersection between $P$ and the band are contained in $W$ (except by a tiny neighborhood of their endpoints). Let $Q=P \cap(M-$ int $W) . Q$ is a planar surface which has several components; some of them are annuli for which one boundary component is in $\partial W$ and the other is a meridian of $\partial \eta(k)$. By eliminating parallel annuli we can suppose that $(Q, \partial Q) \subset$ $(M-$ int $W, \partial M \cup \partial W)$ consist of three components, $Q_{0}, Q_{1}$ and $Q_{\alpha}$ with the following properties:

(a) $Q_{1}\left(Q_{0}\right)$ is an annulus for which one boundary component is $c_{1} \subset \partial W$ $\left(c_{0} \subset \partial W\right)$ and the other is a meridian of $\partial \eta(k)$.

(b) $Q_{\alpha}$ is a connected planar surface, two of its boundary components are meridians in $\partial M$, and the others are parallel to $c_{\alpha}$ in $\partial W$.

According to $\left[\mathrm{Sc}_{3}, 7.1\right], Q$ is a parameterizing surface for $(M, \gamma, \beta)$. Note that $Q$ is incompressible and $\partial$-incompressible in $M$-int $W$. This is clear for $Q_{0}$ and $Q_{1}$. If $Q_{\alpha}$ were compressible then we could make an isotopy in $S^{3}-k_{b}$ to reduce the number of intersections between $P$ and the band. If $Q_{\alpha}$ were $\partial$-compressible, then by looking to the possible types of $\partial$-compression disks and doing an argument similar to $\left[\mathrm{E}_{2}, 2.6\right]$, we could again reduce the number of intersections between $P$ and the band.

1.4. Let $S$ be a $\beta$-taut Seifert surface for $k$; put $S$ in normal position with respect to $Q\left[\mathrm{Sc}_{3}, 7.2\right]$. (See [Sc, 7.7$]$ for the definition of a sutured manifold decomposition respecting a parameterizing surface.) It is not difficult to see and is implicit in [ $\left.\mathrm{Sc}_{3}\right]$ that the sutured manifold decomposition $(M, \gamma) \stackrel{S}{\longrightarrow}\left(M_{1}, \gamma_{1}\right)$ is $\beta$-taut and respects $Q$. Here we use the notion of sutured manifold hierarchy as presented in [ $\left.\mathrm{Sc}_{4}, 2.1\right]$. Construct a $\beta$-taut sutured manifold hierarchy

$$
(M, \gamma, \beta) \stackrel{S_{1}=S}{\longrightarrow}\left(M_{1}, \gamma_{1}, \beta_{1}\right) \stackrel{S_{2}}{\longrightarrow} \cdots \rightarrow\left(M_{n}, \gamma_{n}, \beta_{n}\right)
$$

respecting $Q \quad\left[\mathrm{Sc}_{4}, 2.5\right]$ (see also $\left[\mathrm{Sc}_{3}, 4.19\right.$ and $\left.\mathrm{Sc}_{3}, 7.8\right]$ ). $\partial M_{n}$ is a collection of spheres. $S$ meets $\beta_{0}\left(\beta_{1}\right)$ in one point and $Q_{0}\left(Q_{1}\right)$ in one arc. Following $\left[\mathrm{Sc}_{3}, 2.4(\mathrm{c}), \mathrm{Sc}_{4}, 2.1\right]$ the edges $\beta_{0}, \beta_{1}$ and $\beta_{\alpha}$ can be oriented so that at any point of intersection with an $S_{i}$ (hence with $R\left(\gamma_{i}\right)=R_{+}\left(\gamma_{i}\right) \cup R_{-}\left(\partial_{i}\right)$ ) the orientation points in the direction of the normal vector to $S_{i}$. We can suppose $\beta$ has one of the orientations showed in the Figure 3 (the choice of one of them depends on the orientation of $S_{1}$ ). The argument for the two cases is more or less similar, and we will do both together until $\S 4$, where some differences 


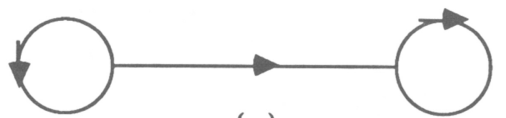

(a)

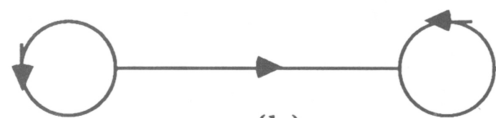

(b)

FIGURE 3

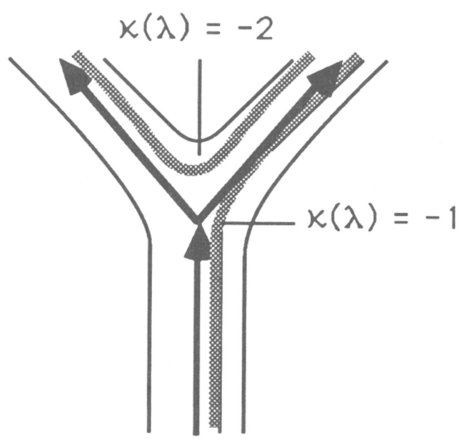

FIGURE 4

arise. Consider $R_{+}\left(\gamma_{i}\right)\left(R_{-}\left(\gamma_{i}\right)\right)$ as the part of $\partial M_{i}$ in which the orientation points out of (into) $M_{i}$. Denote by $Q_{i}$ and $\beta_{i}$ the remnants of $Q$ and $\beta$ in $M_{i}$. Sometimes for simplicity $\beta_{i}, R_{+}\left(\gamma_{i}\right), R_{-}\left(\gamma_{i}\right), R\left(\gamma_{i}\right)$ will be denoted by $\beta, R_{+}, R_{-}, R(\gamma)$ respectively.

1.5. Recall from $\left[\mathrm{Sc}_{3}, 7.4\right]$ what the index of a parameterizing surface is, $I(Q)=$ $\nu+\mu+\mathscr{K}-2 \chi(Q)$, where $\nu$ is the number of sutures and $\mu$ the number of edges that $\partial Q$ crosses. For each arc $\delta$ of $\partial Q \cap \eta(v)$, where $v$ is a vertex of $\beta$, define $\kappa(\delta)$ to be -1 if $\delta$ passes between an edge of $\beta$ pointing into the vertex and one pointing out, and define $\kappa(\delta)=-2$ if $\delta$ passes between edges of $\beta$ both pointing into the vertex or both pointing out (see Figure 4). The curve $c_{\alpha} \subset \partial W$ has $\nu=0, \mu=4$, and $\mathscr{K}=-6$, and the curves $c_{0}, c_{1}$ each have $\nu=0, \mu=1, \kappa=-1$. Hence if $Q_{\alpha}$ has $p$ boundary components in $\partial W, \chi\left(Q_{\alpha}\right)=-p$ and so $I\left(Q_{\alpha}\right)=-2 p+2 p=0$. Also $I\left(Q_{0}\right)=I\left(Q_{1}\right)=0$.

1.6. Suppose that for an arc $\lambda$ of $\beta_{n}$, which contains no vertices, there is a disk $D \subset Q_{n}$, such that $\partial D=\delta_{1} \cup \delta_{2}$, where $\delta_{1} \subset \eta(\lambda), \delta_{2} \subset \partial M_{n}$, and $\delta_{2}$ crosses a suture; $\lambda$ is called a cancelable arc, and $D$ is called a canceling disk. Then there is a product disk $D^{\prime}$ which surrounds $\lambda$, namely $D^{\prime}=\operatorname{cl}\left(\partial \eta(D \cup \lambda)-\partial M_{n}\right)$. The sutured manifold decomposition $\left(M_{n}, \gamma_{n}, \beta_{n}\right) \stackrel{D^{\prime}}{\longrightarrow}\left(M_{n}^{\prime}, \gamma_{n}^{\prime}, \beta_{n}^{\prime}\right)$ is $\beta$-taut [Sc $\left.\mathrm{Sc}_{3}, 4.2\right]$ and it is not difficult to arrange it so that respects $Q_{n}$. Do enough of these operations so that the components of $M_{n}$ which contain the vertices do not contain cancelable arcs. Still denote the last step as $M_{n}$. (Compare to [ $\mathrm{Sc}_{3}$, 4.3] in which a slight different procedure is made to eliminate cancelable arcs.)

1.7. Denote by $Q_{i, n}, i=0,1$, the parameterizing surface obtained from $Q_{i}$ at the end of the hierarchy. Similarly denote by $\beta_{i, n}, i=1,0$, the 1 -complex obtained from $\beta_{i}$ at the end of the hierarchy. The parameterizing surface $Q_{i, n} \subset Q_{i}, i=0,1$, has $I\left(Q_{i, n}\right) \leq 0\left[\mathrm{Sc}_{3}, 7.5,7.6\right]$.

Claim. Each component $q$ of $Q_{i, n}$ for which $\partial Q_{i, n} \cap \eta\left(\beta_{i, n}\right) \neq \varnothing$ is a disk for which $I(q)=0$. Indeed $\partial q \cap \beta_{i, n}$ is a single arc and $\partial q \cap \partial M_{n}$ is a single arc crossing a single suture. 
Proof. This is similar to [ $\mathrm{ST}_{1}$, Claim 2]. Note that the surface $S_{1}$ meets $\beta_{0}$ and $\beta_{1}$.

This claim implies that there is a component $q$ of $Q_{i, n}, q$ in a disk, such that $\partial q$ runs through one of the vertices; all the other components of $Q_{i, n}$ are canceling disks for an arc of $\beta_{i, n}$. So the bits of $\beta_{0}$ and $\beta_{1}$ which do not contain a vertex of $\beta$ can be eliminated as in 1.6, via the remnants of $Q_{i, n}$.

1.8. A component of $M_{n}$ which does not meet $\beta$ is a 3-ball with a single suture in its boundary. We will disregard these trivial components and suppose with no loss of generality that all the components of $M_{n}$ meet $\beta$. As a consequence of this we conclude that every disk component of $R_{ \pm}$intersects $\beta$. Note that a component of $\partial M_{n}$ has sutures, for if there is a sphere $S$ without sutures, it has to meet $\beta$. But $S$ only can meet $\beta_{\alpha}$, for all the arcs of $\beta_{0}$ and $\beta_{1}$ meet components of $\partial M_{n}$ which contain sutures. For $\beta$-tautness $\beta_{\alpha}$ has to meet $S$ always in the same direction, so if $\beta_{\alpha}$ meets $S$ twice or more it will imply that there is a nonseparating sphere in $S^{3}$, which is impossible; if $\beta_{\alpha}$ meet $S$ once then the band has to be trivial, contradicting the hypothesis.

\subsection{Claim. $\chi\left(R_{+}\left(\gamma_{n}\right)\right)=\chi\left(R_{-}\left(\gamma_{n}\right)\right)$.}

Proof. $R_{ \pm}$contains no closed component, so by [Sc $\left.\mathrm{Sc}_{3}, 1.1\right], \chi_{\beta}\left(R_{ \pm}\right)=\mid R_{ \pm} \cap$ $\beta\left|-\chi\left(R_{ \pm}\right)+\right|$disks in $R_{ \pm}$disjoint from $\beta \mid$. By $1.8 R_{+}$and $R_{-}$have the same number of disks disjoint from $\beta$. Also in our case $\left|R_{+} \cap \beta\right|=\left|R_{-} \cap \beta\right|$. By $\beta$-tautness of $\left(M_{n}, \gamma_{n}, \beta_{n}\right), \chi_{\beta}\left(R_{+}\right)=\chi_{\beta}\left(R_{-}\right)$. This implies that $\chi\left(R_{+}\right)=$ $\chi\left(R_{-}\right)$.

1.10 Claim. Either $(M, \gamma)$ satisfies (d) or (e) of Theorem 1 or $\left(M_{n}, \gamma_{n}\right)$ is not $\varnothing$-taut.

Proof. If $\left(M_{n}, \gamma_{n}\right)$ is $\varnothing$-taut then by [ $\left.\mathrm{Sc}_{4}, 2.7\right]\left(M_{i}, \gamma_{i}\right)$ is $\varnothing$-taut for all $i \geq 1$, and either $(M, \gamma)$ is $\varnothing$-taut, and so is irreducible and $\partial$-incompressible or $M$ is a solid torus (i.e. $k$ is the trivial knot). Now in any case, by [ $\left[\mathrm{Sc}_{3}, 3.3\right] S$ is $\varnothing$-taut. This implies (d) or (e) of Theorem 1.

So in what follows we made the following

\subsection{Assumption. $\left(M_{n}, \gamma_{n}\right)$ is not $\varnothing$-taut.}

1.12. $\partial M_{n}$ is a collection of spheres, so $\left(M_{n}, \gamma_{n}\right)$ is $\varnothing$-taut if and only if each sphere has only one suture and it is the boundary of a 3-ball. Note that if a component of $M_{n}$ has only one sphere in its boundary, then this sphere bounds a 3-ball, for $M_{n}$ is contained in $S^{3}$.

\subsection{Claim. Some of the surfaces $S_{i}$ intersect $\beta_{\alpha}$.}

Proof. Suppose that none of the $S_{i}$ 's meets $\beta_{\alpha}$.

Case 1. The boundary of the component of $M_{n}$ which contains $\beta_{\alpha}$ is a sphere, say $T$.

By $1.7,1.8$ there are only two disks components in $R(\gamma)$. If there is only one suture, then $\left(M_{n}, \gamma_{n}\right)$ is $\varnothing$-taut, which contradicts 1.11 . So $T$ contains more than one suture. Then $R(\gamma)$ consist of two disks and a collection of annuli. By $1.9 \chi\left(R_{+}\right)=\chi\left(R_{-}\right)$, so it follows that $R_{+}\left(R_{-}\right)$consist of one disk and $m$ 

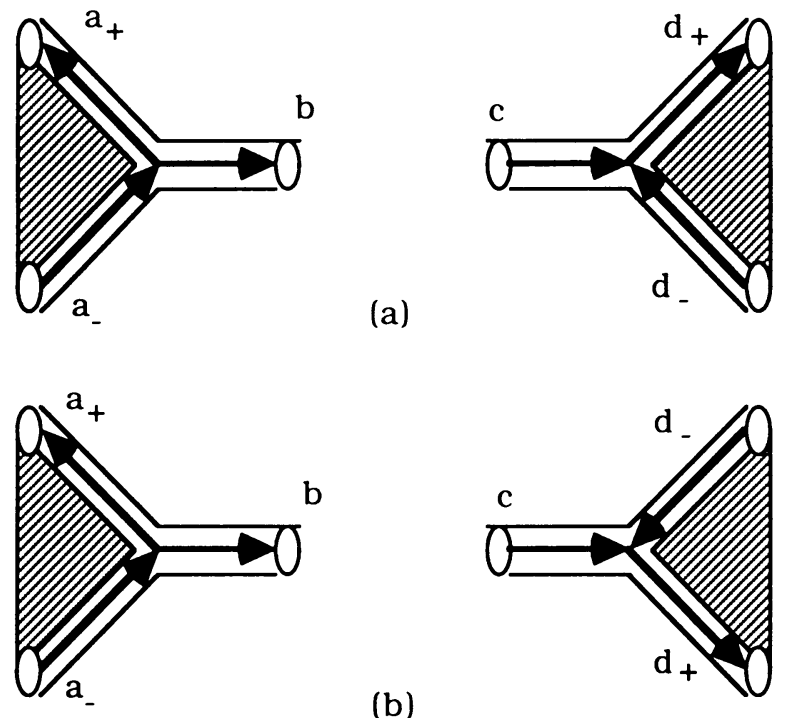

(b)

FIGURE 5

annuli. Then the innermost annulus $A$ of $R_{-}$must have $\beta$-norm equal to 1 , because it meets $\beta$ once. Using the component $q$ of $Q_{i, n}$ described in 1.7, it is not difficult to see that there are two disks $D_{1}, D_{2}$ (take disks parallel to the innermost disk in $\left.R_{+}\right)$such that $\partial\left(D_{1} \cup D_{2}\right)=\partial A,\left[D_{1} \cup D_{2}\right]=[A]$ in $H_{2}\left(M_{n}, \chi(\partial A)\right)$, and each one meets $\beta$ once. Then $\chi_{\beta}\left(D_{1} \cup D_{2}\right)=0$, but that implies that $R_{-}$is not $\beta$-taut.

Case 2. $\partial M_{n}$ has two components, say two spheres $T_{1}, T_{2}$. A sphere parallel to $T_{1}$ meets $\beta_{\alpha}$ in one point, implying that the band is trivial.

1.14. Following 1.13 there are two components of $\beta_{n}$ which have a vertex. Denote the component which has two ends in $R_{+}\left(R_{-}\right)$and one in $R_{-}\left(R_{+}\right)$ by $A_{+}\left(A_{-}\right)$. Denote the ends of $A_{+}$by $a_{+}, a_{-}, b$, and the ends of $A_{-}$by $d_{+}, d_{-}, c$, where $b, c$ are the ends which are part of $\beta_{\alpha} . a_{+}, d_{+}, b$ lie on $R_{+}$, and $a_{-}, d_{-}, c$ lie on $R_{-} . a_{+}, a_{-}\left(d_{+}, d_{-}\right)$are part of $\beta_{0}\left(\beta_{1}\right) .1 .7$ shows that $a_{+}$and $a_{-}\left(d_{+}\right.$and $\left.d_{-}\right)$lie in adjacent components of $R_{ \pm}$and there is a disk $q$ component of $Q_{0, n}\left(Q_{1, n}\right)$ such that $\partial q \cap \partial M_{n}$ is an arc joining $a_{+}$and $a_{-}\left(d_{+}\right.$and $\left.d_{-}\right)$which crosses a suture. See Figure 5.

There is a collection of arcs on $A_{+}$going from $a_{+}$to $b$; each such arc $\lambda$ is contained in the boundary of a component of $Q_{n}$, and for this arc $\kappa(\lambda)=$ -2 . Call the part of $A_{+}$which contains these arcs the negative side of $A_{+}$. Analogously, there is a collection of arcs on $A_{+}$going from $a_{-}$to $b$. For each such arc $\lambda, \kappa(\lambda)=-1$. Call the part of $A_{+}$which contains these arcs the positive side of $A_{+}$. In a similar way we define the negative and positive side of $A_{-}$.

\subsection{Claim. No component of $Q_{n}$ has negative index.}

Proof. If $q$ is a component of $Q_{n}$ with $I(q)=\nu+\mu+\mathscr{K}-2 \chi(q)<0$, then because $\nu+\mu+\mathscr{K} \geq 0$, it follows that $\chi(q)>0$, so $q$ is a disk, $\nu+\mu+\mathscr{K}=0$, $I(q)=-2$, and then $\partial q$ crosses no suture, and the only arcs of $\beta$ that it can cross are the negative side of $A_{+}$or $A_{-}$(it cannot cross both of them 
because then $\partial q$ would cross sutures or other arcs). So $\partial q$ consist of arcs which are contained in $R_{+}$, say, and arcs over $A_{+}$which go from $a_{+}$to $b$. An application of [ $\left.\mathrm{Sc}_{3}, 6.2\right]$ shows that either there are compressible $\beta$-loops [ $\left.\mathrm{Sc}_{3}, 6.1\right]$ for $Q_{\alpha}$ in $a_{+}$or $b$, or there is a sphere intersecting $\beta$ in one point, or $\partial q \subset R_{+}$. The first contradicts the $\partial$-incompressibility of $Q_{\alpha}$, the second implies that the band is trivial, and the third contradicts the fact that $q$ is part of a parameterizing surface.

1.16 Claim. Each component of $Q_{n}$ is a disk or annulus of index zero.

Proof. $I\left(Q_{n}\right) \leq I(Q)=0$ and $Q_{n}$ has no negative index components, then $I\left(Q_{n}\right)=0$, and each component of $Q_{n}$ has index zero. For $q$ a component of $Q_{n}, I(q)=\nu+\mu+\mathscr{K}-2 \chi(q)=0$, but $\nu+\mu+\mathscr{K} \geq 0$, so $\chi(q) \geq 0$, and then $q$ is a disk or annulus.

Note that if $q$ is an annulus component of $Q_{n}$, then $\partial q$ crosses no suture, and the only arcs of $\beta$ that it can cross are the negative side of $A_{+}$or $A_{-}$.

The following lemma will avoid possible technical problems in $\S 4$.

1.17 Lemma. It can be supposed that in each step of the sutured manifold hierarchy $Q_{i}=Q_{i-1}-$ int $\eta\left(S_{i}\right)$.

Proof. According to $\left[\mathrm{Sc}_{3}, 7.5,7.6\right]$ that is the case except possibly when a product disk, product annulus or a disk with boundary in $R(\gamma)$ was used in the hierarchy. In [ $\left.\mathrm{Sc}_{3}, 7.6\right]$ is explained what changes are made to the parameterizing surface in that case, but in general these changes decrease the index. Let $S_{i}$ be the first of such surfaces used in the hierarchy. If $I(Q)$ does not drop then either there are no arcs of intersection between $S_{i}$ and $Q_{i-1}$ whose two ends lie in $R_{+}\left(R_{-}\right)$, or each such arc bounds a disk in $Q_{i-1}$ which crosses no suture and no arc of $\beta$. In the last case $S_{i}$ can be isotoped to not intersect $Q_{i-1}$. After this move the manifold $M_{i}$ looks the same as if we had made the changes mentioned in [ $\left.\mathrm{Sc}_{3}, 7.6\right]$. Do the same to each of these surfaces.

This observation could be made for any $\beta$-taut sutured manifold hierarchy in which the index of the parameterizing surface does not decrease.

\section{THE COMBINATORIAL SETTING}

2.1. Let $T$ be one of the sphere components of $\partial M_{n}$. The points $\beta \cap T$ and the arcs $\partial Q_{n} \cap T$ can be regarded as a graph $\Gamma$ in $T$. A vertex of $\Gamma$ is a point of $\beta \cap T$ and an edge is an arc component of $\partial Q_{n} \cap T$, each one of its ends is at a vertex. Denote the components of $\partial Q_{\alpha}-\partial M$ by $a_{1}, a_{2}, \ldots, a_{p}$, labeled so that $a_{i}$ and $a_{i+1}$ bound an annulus in $\partial W$ disjoint from $Q_{\alpha}$, for $1 \leq i \leq p-1$. Denote by $\varepsilon_{a}=\partial Q_{0, n} \cap T \quad\left(\varepsilon_{d}=\partial Q_{1, n} \cap T\right)$ the edge joining $a_{+}$and $a_{-}\left(d_{+}\right.$and $\left.d_{-}\right)$, this edge exists for $1.7,1.14$; it crosses a suture. Label the end of an edge in $\Gamma$, other than $\varepsilon_{a}$ and $\varepsilon_{d}$, with $i\left(i^{*}\right)$ if this point is over $a_{i} \cap \beta$ in the top (bottom) part of $\beta$ (top and bottom according to Figures 3 and 5). The collection of labels around a vertex in $\Gamma$, other than $a_{ \pm}, d_{ \pm}$, looks like $1,2, \ldots, p, p^{*}, \ldots, 1^{*}$; the collection of labels around $a_{ \pm}, d_{ \pm}$looks like $1,2, \ldots, p$ or $1^{*}, 2^{*}, \ldots, p^{*}$. An edge is level if their ends are equally labeled (ignoring the asterisk). $a_{+}, a_{-}, b, c, d_{+}, d_{-}$are named special vertices, any other is called a simple vertex. Call the part of a special vertex which is contained in the negative (positive) side of $A_{+}$or 
$A_{-}$the negative (positive) side of such vertex (see 1.14). $b$ and $c$ have both negative and positive sides, $a_{+}$and $d_{-}$have only negative side, $a_{-}$and $d_{+}$ have only positive side.

Give an orientation to $Q_{\alpha}$; this orientation induces an orientation in the boundary components of $Q_{\alpha}$. Two components of $\partial Q_{\alpha}-\partial M$ are parallel if with the induced orientation they are homologous in $\partial W$, otherwise they are antiparallel. Put an orientation on $T$, for example the orientation induced by $R_{+}$, this induces an orientation on the vertices of $\Gamma$. Let $v_{1}, v_{2}$ be two vertices of $\Gamma$, other than $a_{ \pm}, d_{ \pm} . v_{1}$ and $v_{2}$ are parallel if with the induced orientation they are homologous on $\partial \eta\left(\beta_{\alpha}\right) \cap \partial W$, otherwise they are antiparallel. $v_{1}$ is parallel to $a_{+}$or $a_{-}$if $v_{1}$ is homologous to $a_{+} \cup a_{-}$in $\partial \eta\left(\beta_{\alpha} \cup A_{+}\right) \cap$ $\partial W$. Analogously for $d_{+}, d_{-} . a_{ \pm}$is antiparallel to $d_{ \pm}$for $a_{+} \cup a_{-}$is not homologous to $d_{+} \cup d_{-}$in $\partial \eta\left(A_{+} \cup \beta_{\alpha} \cup A_{-}\right) \cap \partial W$. Then all the vertices in $R_{+}\left(R_{-}\right)$with the exception of $a_{+}\left(d_{-}\right)$are parallel. A vertex in $R_{+}$is antiparallel to a vertex in $R_{-}$(other than $a_{+}, d_{-}$). We have the following parity rule $\left[\mathrm{Sc}_{1}, 2.2\right]$. Let $v_{1}, v_{2}$ be vertices of $\Gamma, i, j \in\{1, \ldots, p\}$.

(1) If an edge joins parallel vertices $v_{1}$ and $v_{2}$ with labels $i$ and $j$ ( $i$ and $j^{*}$, or $i^{*}$ and $j^{*}$ ) respectively, then $a_{i}$ and $a_{j}$ are antiparallel (resp. parallel, resp. antiparallel).

(2) If an edge joins antiparallel vertices $v_{1}$ and $v_{2}$ with labels $i$ and $j$ ( $i$ and $j^{*}$, or $i^{*}$ and $j^{*}$ ) respectively, then $a_{i}$ and $a_{j}$ are parallel (resp. antiparallel, resp. parallel).

2.2. Let $\Gamma$ be a subgraph of $\Gamma$, and let $x \in\left\{1,2, \ldots, p, 1^{*}, \ldots, p^{*}\right\}$. We say that $\Lambda$ satisfies condition $P(x)$ if (see [CGLS, 2.6]):

For each vertex $y$ of $\Lambda$ there exist an edge of $\Lambda$ incident to $y$ with label $x$, connecting $y$ to a parallel vertex.

For $x \in\left\{1, \ldots, p^{*}\right\}$ an $x$-path is a path in $\Gamma$ so that the beginning point of each edge is labeled with $x$, and all its vertices are parallel. An $x$-cycle is an $x$-path which is a cycle. A Scharlemann cycle is an $x$-cycle which is the boundary of a disk without edges nor vertices in its interior. If a subgraph $\Lambda$ of $\Gamma$ satisfies $P(x)$ for some $x$, then it is possible to construct an $x$-path beginning at a vertex, and then this will form an $x$-cycle in $\Lambda$.

2.3 Lemma. Let $\Lambda$ be a subgraph of $\Gamma$ which consist of the intersection of $\Gamma$ with a disk, and such that all its vertices are parallel, and that satisfies condition $P(x)$ for some $x$, then $\Lambda$ contains a Scharlemann cycle.

Proof. It is a straightforward modification of [CGLS, 2.6.1, 2.6.2], or [BE, 4.4].

2.4 Claim. There is no Scharlemann cycle in $\Gamma$.

Proof. If $\Gamma$ contains a Scharlemann cycle, then either is a loop, which contradicts the $\partial$-incompressibility of $Q_{\alpha}$, or it has at least two edges, and then a modification of $\left[\mathrm{Sc}_{2}, 5.6\right]$ shows that there is a lens space summand in $S^{3}$. One of these Scharlemann cycles may be a cycle in which every edge is labeled with 1 and $1^{*}$ (or $n$ and $n^{*}$ ), i.e. a pure level circuit in the terminology of [BE], but in contrast with the argument there, here a pure level circuit can be used to construct a lens space because all its vertices are parallel.

2.3 and 2.4 imply that there is no subgraph of $\Gamma$ consisting of the intersection of $\Gamma$ with a disk, satisfying $P(x)$ and with all its vertices being parallel. 
2.5 Lemma. Suppose that $D$ is a disk component of $R(\gamma)$ which does not contain $a_{ \pm}$nor $d_{ \pm}$. Then for each $x \in\left\{1, \ldots, p^{*}\right\}$ there is at least one edge with label $x$ in $D$ which crosses the suture surrounding $D$. So there are at least $2 p$ arcs crossing that suture.

Proof. If this does not happen for some $x$ then $\Lambda=D \cap \Gamma$ has $P(x)$, contradicting 2.3, 2.4.

2.6 Lemma. If a disk $D$ of $R_{+}\left(R_{-}\right)$contains only one of $a_{+}, d_{+}\left(a_{-}, d_{-}\right)$, then there are at least $p$ edges, other than $\varepsilon_{a}, \varepsilon_{d}$, crossing the suture surrounding $D$.

Proof. If $a_{-}$or $d_{+}$are in $D$, then the proof is as in 2.5 , for they are parallel to all the other vertices in $D$. If $a_{+}\left(d_{-}\right)$is in $D$, and there are no more vertices there then the conclusion is obvious. If there are more vertices in $D$ then there are at least $p$ labels such that no edge adjacent to $a_{+}\left(d_{-}\right)$has one of these labels in its other end, now do for these labels the same argument as in 2.5.

The following two lemmas will be applied in 3.7, 3.11, 4.1, 4.2, 4.8.

Let $D$ be a disk contained in $T$ so that $\partial D$ cut transversally two special vertices, and so that either the positive or negative part of such vertices is in $D$. Suppose also that all the vertices in the interior of $D$ are simple, and all the vertices in $D$ are parallel, including the special vertices in the boundary. Suppose that no edge crosses $\partial D$. Let $\Lambda=\Gamma \cap D$. Call the labels of the two special vertices which meet $\partial D$, the labels of $\partial D$.

2.7 Lemma. Suppose $\partial D$ is labeled with $\left\{1, \ldots, p, p^{*}, \ldots, 1^{*}\right\}$, in this order, and $\Lambda$ looks like Figure 6 . Then either there is a Scharlemann cycle which intersects at most one side of $\partial D$, or for each $i \in\{1, \ldots, p\}$ there is an i-path beginning at one side of $\partial D$ and finishing in the other side of $\partial D$ with label $i^{*}$. Furthermore all the edges adjacent to $\partial D$ have ends labeled $i-i^{*}$, i.e. they are level.

Proof. Suppose there is no such Scharlemann cycle. For the label 1, take a 1-path $\gamma_{1}$ beginning at $\partial D$, this path has to finish at the other side of $\partial D$, otherwise there is a 1-cycle (and by 2.3 a Scharlemann cycle); so it finishes at $i_{1}^{*} \quad\left(i_{1}^{*} \geq 1\right)$. The complementary part of $\gamma_{1}$ which contains the other labels $j$ 's of $\partial D$ has $P(2)$, because all the vertices are parallel. Take now a

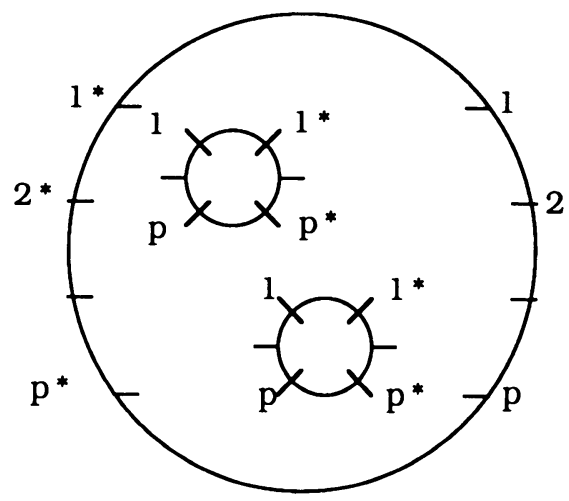

FIGURE 6 


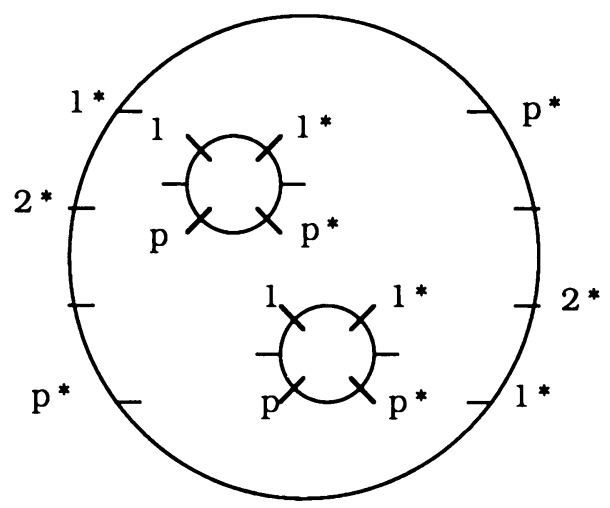

Figure 7

2-path $\gamma_{2}$ beginning at $\partial D$, it finishes at $\partial D$ with label $i_{2}^{*}, i_{2}^{*}>i_{1}^{*}$, and the complementary part of $\gamma_{2}$ has $P(3)$. So by induction we can take a $k$-path $\gamma_{k}$ beginning at $\partial D$, which finishes at $\partial D$ with label $i_{k}^{*}, i_{k}^{*}>i_{t}^{*}$ if $k>t$, and the complementary region of $\gamma_{k}$ has $P(k+1)$. This implies $i_{k}^{*}=k^{*}$ for all $k$, so the last edge of each of these paths has labels $k-k^{*}$. Repeating the argument, but now beginning the paths at $i^{*}$ in $\partial D$, one conclude that the edges of $\partial D$ with label $i$, have the other end with label $i^{*}$.

2.8 Lemma. Suppose $\partial D$ is labeled with $\left\{1^{*}, \ldots, p^{*}, 1^{*}, \ldots, p^{*}\right\}$, in this order (or $\{1, \ldots, p, 1, \ldots, p\}$ ), and $\Lambda$ looks like in Figure 7. Then there is a Scharlemann cycle in $D$; if this cycle contains edges which meet $\partial D$ then the cycle does not contain the part of $\partial D$ between the labels $1^{*}$ and $p^{*}(1$ and $p)$.

Proof. Do the same as in 2.7 , construct the paths $\gamma_{k^{*}}$. After reaching the other end of $\partial D$ this path can be continued implying the existence of a $k^{*}$-cycle, and hence a Scharlemann cycle, unless this path ends with an edge labeled $k^{*}-k^{*}$ which is not possible for it contradicts the parity rule.

\section{Possible CONfigurations of $\left(M_{n}, \gamma_{n}, \beta_{n}\right)$}

The goal of this and next section is to show that there are two vertices of $\Gamma$ joined by $p$ parallel level edges, one for each label, and such that these edges when viewed in $Q$ are bad loops (see 4.3). In this section we prove that each sphere of $\partial M_{n}$ has at most two disks components of $R(\gamma)$ and list a finite number of possible configurations of the arcs of $\beta$ in $M_{n}$. In this and subsequent sections $R(\gamma), R_{+}, R_{-}$will denote $R\left(\gamma_{n}\right), R_{+}\left(\gamma_{n}\right), R_{-}\left(\gamma_{n}\right)$ respectively.

3.1 Claim. A component $N$ of $M_{n}$ which does not contain a vertex of $\beta$ is a 3-ball with one suture on its boundary, so is $\varnothing$-taut.

Proof. The proof is by induction on the number $r$ of arcs of $\beta$ that are contained in $N$. If $r=0$ then by $\beta$-tautness $N$ is as required. So suppose $r>0$. Note that by 1.8 each component of $\partial N$ has sutures. There are two possibilities:

(a) There is in $N$ an arc $\beta^{\prime}$ which is part of $\beta_{0}$ or $\beta_{1}$. Then $\beta^{\prime}$ is a cancelling arc via $Q_{0}$ or $Q_{1}$, and doing the operation described in 1.6, we get 
$N^{\prime}$, which contains $r-1$ arcs of $\beta$. By induction $N^{\prime}$ is as required, and then it is not difficult to see that $N$ is also a 3-ball and is $\varnothing$-taut.

(b) All the arcs in $N$ are part of $\beta_{\alpha}$. Let $D$ be a disk in $R(\gamma)$; by 2.5 there is at least one edge crossing the boundary of $D$, this edge has to be part of an index zero disk $F$, and as $F$ does not meet the vertices of $\beta, \partial F$ crosses a suture and goes through an arc $\beta^{\prime}$ of $\beta$, so $F$ is a cancelling disk for $\beta^{\prime}$. Now proceed as in case (a).

We are assuming that $M_{n}$ is not $\varnothing$-taut (1.11), and so by 3.1 we can assume now that all the components of $M_{n}$ contain a vertex of $\beta$.

3.2 Claim. A disk $D$ in $R_{ \pm}$must contain special vertices.

Proof. Suppose this is false. Suppose w.l.o.g. that $D$ is in $R_{+}$. By 2.5 there are at least $2 p$ edges crossing the suture $\partial D$. Each of these edges belong to an index zero disk, and then these edges have to meet $c$ or $d_{-}$in its negative part, otherwise these disks would be cancelling disks. But there are only $p$ negative labels in $c$, and if a disk meet one of these labels it also meets the corresponding label in $d_{-}$, so in $c \cup d_{-}$there are room only for $p$ edges coming from $D$, so the other $p$ edges have to be part of cancelling disks, which is a contradiction.

Claim 3.2 implies that a component of $\partial M_{n}$ has at most four innermost sutures, i.e. sutures which bound a disk contained in $R_{ \pm}$, since $a_{ \pm}\left(d_{ \pm}\right)$lie in adjacent components of $R_{ \pm}$.

3.3 Claim. If a disk $D$ in $R_{ \pm}$contains a special vertex, then the component adjacent to it also has a special vertex.

Proof. This is clear if $D$ contains one of $a_{+}, a_{-}, d_{+}, d_{-}$. So suppose that $D$ contains $b$ and no other special vertex. By 2.5 there are at least $2 p$ edges which cross the suture $\partial D$. At most $p$ edges come from the negative side of $b$, so at least $p$ edges come from a simple vertex or the positive side of $b$. If an edge come from the positive side of $b$, its other end meets either $a_{-}$or the negative side of a special vertex, and if the edge come from a simple vertex then its other end has to meet the negative side of a vertex, so there is a special vertex in the component adjacent to $D$.

\subsection{Claim. A disk $D$ in $R_{ \pm}$contains at most two special vertices.}

Proof. Suppose $D$ contains three special vertices, say $a_{+}, d_{+}$and $b$. The component $E$ adjacent to $D$ will contain $a_{+}$and $d_{-}$. If $c$ is in $E$, then by 3.2 there are no more sutures, and then $E$ is a disk, so $M_{n}$ is a 3-ball with a suture in its boundary, and so is $\varnothing$-taut by 1.12 , which contradicts Assumption 1.11. If $c$ is not in $E$, then there is another disk $D^{\prime}$ such that $c$ is in $D^{\prime} . D^{\prime}$ cannot be adjacent to $E$, since both are in $R_{-}$, so in the area adjacent to $D^{\prime}$ there is no special vertex which contradicts 3.3.

3.5 Claim. A component $T$ of $\partial M_{n}$ has at most three disks components of $R(\gamma)$.

Proof. By 3.2 $T$ has at most four innermost sutures. So suppose $T$ has four disks of $R(\gamma)$, call them $D_{1}, D_{2}, D_{3}, D_{4}$. Then, say, $b$ is in $D_{1}, c$ in $D_{2}$, $a_{ \pm}$in $D_{3}, d_{ \pm}$in $D_{4}$. Let $E_{-}\left(E_{+}\right)$be the area adjacent to $D_{1}\left(D_{2}\right)$, it contains special vertices by 3.3 . Then $E_{-}\left(E_{+}\right)$contains either $a_{-}$or $d_{-}\left(a_{+}\right.$or $\left.d_{+}\right)$ 


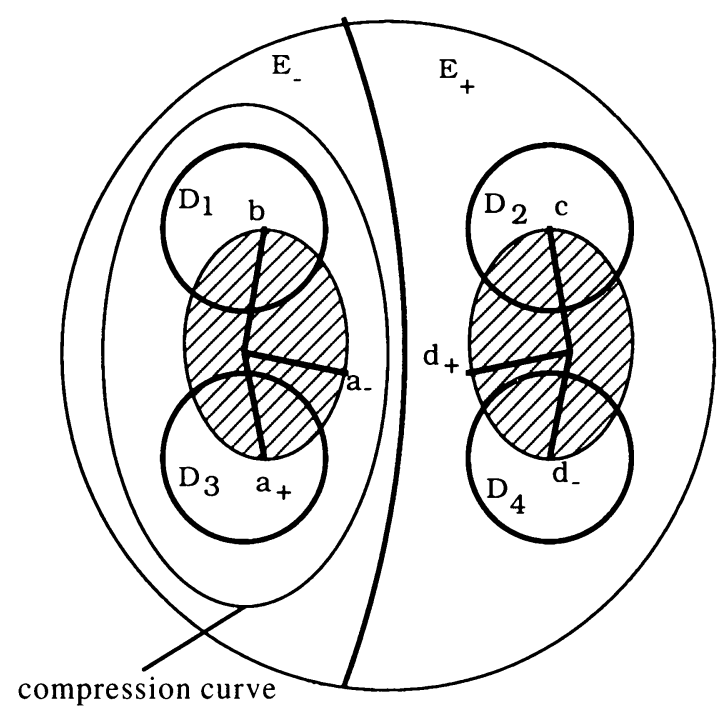

FIGURE 8

and must be adjacent to two disks components of $R_{+}\left(R_{-}\right)$. It follows that $E_{-}, E_{+}$are three-punctured spheres.

Case 1. $d_{-}$is in $E_{-}$.

Then $d_{+}$is in $D_{4}$, and $E_{-}$is adjacent to $D_{4}$. There are, by 2.5 , at least $2 p$ edges which cross $\partial D_{1}$, and $p$ of them have to meet $d_{-}$, for only $p$ may come from the negative side of $b$. By 2.6 there are $p$ edges which cross $\partial D_{4}$, and they have to meet a special vertex; but there is no special vertex in $E_{-}$ other than $d_{-}$, and there is no place in $d_{-}$for these $p$ edges. So this case it is not possible.

Case 2. $a_{-}$is in $E_{-}$.

Then $a_{+}$is in $D_{3}$, and $E_{-}$is adjacent to $D_{3}$. There are $2 p$ edges which cross $\partial D_{1}$. If they come from simple vertices, then they must meet the negative side of a vertex, but there is no such vertex in $E_{-}$. Then the $2 p$ edges come from $b ; p$ of them have to join the positive side of $b$ and $a_{-}$. By 2.6 there are $p$ edges which cross $\partial D_{3}$, they cannot come from simple vertices, so the only possibility left is that these edges come from $a_{+}$, cross $\partial D_{3}, \partial D_{1}$ and then meet the negative side of $b$. This argument implies that there are no simple vertices in $D_{1}$ and $D_{3}$. A similar situation happens in $D_{2}, D_{4}$ and $E_{+}$. We have a situation as in Figure 8 ; now it is not difficult to see that $E_{-}$is then compressible, which contradicts $\beta$-tautness.

3.6 Lemma. Let $D$ be a disk in $R_{ \pm}$. Suppose that the area adjacent to $D$ is an annulus $E$. Suppose $a_{+}\left(d_{-}\right)$is the only special vertex in $D$. Then $D$ contains simple vertices and $E$ contains either $c$ or $d_{-}\left(\begin{array}{lll}b & \text { or } a_{+}\end{array}\right)$.

Proof. $a_{-}\left(d_{+}\right)$is in $E$.

Claim 1. There are simple vertices in $D$.

If $a_{+}$is the only vertex in $D$, then $E$ is not $\beta$-taut. To see that consider two disks $D_{1}, D_{2}$, so that $\partial D_{1} \cup \partial D_{2}=\partial E$, and $[E]=\left[D_{1} \cup D_{2}\right]$, these disks can be chosen so that $D_{1}$ meets $\beta$ in one point and $D_{2}$ meets $\beta$ in as many points 
as $E$ does, say $n$. Then $\chi_{\beta}(E)=n$, but $\chi_{\beta}\left(D_{1} \cup D_{2}\right)=n-1$. This contradicts the $\beta$-tautness of $E$. Note that this construction can be done because of the existence of a disk in $Q_{0, n}$ whose boundary go through $a_{+}, a_{-}$and crosses a suture (cf. 1.7).

Claim 2. No edge adjacent to $a_{+}$crosses $\partial D$ (other than $\varepsilon_{a}$ ).

Suppose an edge $\eta$ adjacent to $a_{+}$crosses $\partial D$. At most $p-1$ edges adjacent to $a_{+}$meet simple vertices in $D$, so there are $p+1$ labels which are not the other end of an edge adjacent to $a_{+}$; for each one of these labels we can find an edge which crosses $\partial D$ (the argument is similar to that of 2.6) and then there will be at least $p+1$ edges coming from simple vertices which cross $\partial D$. These edges have to meet the negative side of a vertex, but in the negative side of a vertex there is room only for $p$ edges, so this is not possible.

Then the $p$ edges which cross $\partial D$ come from simple vertices and have to meet the negative side of a vertex, the lemma follows.

3.7 Lemma. Let $D$ be a disk in $R_{ \pm}$. Suppose that the area adjacent to $D$ is an annulus $E$. Then $a_{-}\left(d_{+}\right)$cannot be the only special vertex in $D$.

Proof. Suppose $a_{-}$is the only special vertex in $D$. Then $a_{+}$is in $E$. $D$ contains simple vertices and no edge adjacent to $a_{-}$crosses $\partial D$ (other than $\varepsilon_{a}$ ). The proof is similar to that in 3.6. By 2.6, and because at a special vertex there is room only for $p$ edges, there are exactly $p$ edges which cross $\partial D$ (other than $\varepsilon_{a}$ ).

Claim. The $p$ edges which cross $\partial D$ meet the negative side of $b$.

The other possibility for these edges is that they meet $a_{+}$. For each $i$, $i=1, \ldots, p$, take a $i^{*}$-path $\lambda_{i}$ starting at $a_{-}$. Each path has to cross $\partial D$, and then meet $b$ or $a_{+}$. This produce the $p$ edges which cross $\partial D$. So suppose that for some $i, \lambda_{i}$ finishes at $a_{+} \cdot \lambda_{i}$ is an $i^{*}$-path in $D \cup E$ which starts in $a_{-}$and finishes in $a_{+}$, and so that $\lambda_{i} \cup \varepsilon_{a}$ bound a disk $D^{\prime} \subset D \cup E . d_{+}$is not in $D^{\prime}$, for then $d_{-}$would be in $D$. There is no other vertex in the interior of $E \cap D^{\prime}$, for if there are vertices, less than $p$ edges join $a_{+}$and these vertices. So there are more than $p$ labels $j$ in these vertices, at which no edge coming from $a_{+}$is incident, so for each such label there is a $j$-path in $E \cap D^{\prime}$ which has to cross the part of $\partial D$ lying in $D^{\prime}$, that is, there are more than $p$ edges crossing $\partial D$, but this is not possible. Either the label $(i-1)^{*}$ or $(i+1)^{*}$ at $a_{-}$lie in $D^{\prime}$, say the label $(i-1)^{*}, i$ as above (possibly $1^{*}=i^{*}$ ). It is not difficult to see that $\lambda_{i-1}$ lies in $D^{\prime}$, and so it finishes at $a_{+}$. Continuing in this way as in the proof of 2.7, we conclude that either there is a Scharlemann cycle in $D$, or $\lambda_{1}$ consists of level edges, starts at $a_{-}$and finishes at $a_{+}$, and $\lambda_{1} \cup \varepsilon_{a}$ bound a disk $D^{\prime \prime}$ with interior disjoint from $\Gamma$. $D^{\prime \prime}$ can be used, as in 2.4 , to construct a lens space in $S^{3}$, which is impossible. So we conclude that the $p$ edges which cross the suture $\partial D$ meet the negative side of $b$.

If $p=1$ there is an edge with label $1^{*}$ at a simple vertex in $D$ and label 1 at $b$, which contradicts the parity rule. So suppose $p>1$. Let $\eta_{1}\left(\eta_{p}\right)=$ (edge adjacent to $b$ at label $1(p)) \cap E$, and let $\alpha$ be the arc in $b$, which go through the negative side of $b$, and which joins the labels 1 and $p$. Then $\eta_{1} \cup \eta_{p} \cup \alpha$ $\cup$ (arc on $\partial D$ joining $\eta_{1}$ and $\eta_{p}$ ) bounds a disk $F \subset E$. We have two cases: Case 1. The positive side of $b$ is not in the interior of $F$.

Then all the edges adjacent to the negative side of $b$ lie in $F$. No vertex is in the interior of $F$, for if there is one, then by 2.3 we could find a 
Scharlemann cycle inside $F$. The negative side of $b$ has labels $1,2, \ldots, p$, and $b$ is antiparallel to all vertices in $D$. Relabel the negative side of $b$ by $1^{*}, 2^{*}, \ldots, p^{*}$; with the new labeling the negative side of $b$ appears as a vertex parallel to all the vertices in $D$. Note that the parity rule still holds under the new labelling. Now an application of 2.8 -where $a_{-}$and the negative side of $b$ are considered the boundary vertex-yields a contradiction.

Case 2. The positive side of $b$ is in the interior of $F$.

Then no edge adjacent to the negative side of $b$ is in the interior of $F \cdot a_{+}$ must lie in $F$, for otherwise as the positive side of $b$ is in $F$, we could find a Scharlemann cycle in $F . a_{+}$has labels $1,2, \ldots, p$, and it is antiparallel to all vertices in $F$. Relabel $a_{+}$by $1^{*}, 2^{*}, \ldots, p^{*}$; with the new labeling $a_{+}$ appears as a vertex parallel to all the vertices in $F$. Now an application of $2.8-$ where $a_{+}$and the positive side of $b$ are considered the boundary vertex-yields a contradiction.

3.8 Lemma. A component $T$ of $\partial M_{n}$ has at most two innermost sutures.

Proof. By 3.5 a component $T$ of $\partial M_{n}$ has at most three innermost sutures, so suppose $T$ has three disks $D_{1}, D_{2}, D_{3}$ components of $R(\gamma)$. The other components of $R(\gamma)$ are annuli and a three punctured sphere $F$. By 1.9 $\chi\left(R_{+}\right)=\chi\left(R_{-}\right)$, so necessarily the disks $D_{1}, D_{2}$ and $F$ belong to the same part of $R(\gamma), R_{+}$or $R_{-}$, say $R_{+}$, and the remaining disk $D_{3}$ belong to $R_{-}$. Then $F$ cannot be adjacent to the disks $D_{i}, i=1,2$. The area adjacent to $D_{1}$ and $D_{2}$ has to consist of annuli. We can assume that $b$ is in $D_{1}$ or $D_{2}$, for otherwise $d_{+}$would be the only special vertex in one of $D_{1}$ or $D_{2}$ contradicting 3.7. So without loss assume that $b$ is in $D_{1}$. If $D_{2}$ contains both special vertices $a_{+}$and $d_{+}$, then $c$ is in $D_{3}$, and there will not be a special vertex in the area adjacent to $D_{3}$, contradicting 3.3. So by $3.7 D_{2}$ contains only $a_{+}$, and $a_{-}$is in the adjacent annulus.

Case 1. $c$ is in $D_{3}$.

Then by 3.6 the annulus adjacent to $D_{2}$ contains $d_{-}$as well as $a_{-}$. Then the annulus adjacent to $D_{1}$ could contain no special vertex, again contradicting 3.3.

Case 2. $c$ is not in $D_{3}$.

Then $d_{-}$is in $D_{3}$ and by $3.6 c$ is in the annulus adjacent to $D_{2}$. Again there is no special vertex in the annulus adjacent to $D_{1}$.

3.9 Lemma. Let $D$ be a disk in $R_{ \pm}$. Suppose that the area adjacent to $D$ is an annulus $E$. Suppose $a_{-}\left(d_{+}\right)$is in $E$, and $b(c)$ is the only special vertex in $D$. Then either $c$ or $d_{-}\left(b\right.$ or $\left.a_{+}\right)$are in $E$.

Proof. Suppose $a_{-}$is the only special vertex in $E$. There are $2 p$ edges which cross the suture $\partial D$, none can come from a simple vertex, for there is no special vertex with negative side in $E$, so the $2 p$ edges crossing $\partial D$ are adjacent to $b$. It follows that there is no simple vertex in $D$. The $p$ edges adjacent to the positive side of $b$ have to meet $a_{-}$. There is an index zero disk $q$, with $\partial q$ running from $a_{-}$to $b$ along $\beta$ and then running across a suture. Then using $q$ (as in 3.6, Claim 1), we can find two disks $D_{1}, D_{2}$, so that $\partial D_{1} \cup \partial D_{2}=\partial E$, and $[E]=\left[D_{1} \cup D_{2}\right]$. These disks can be chosen so that $D_{1}$ meets $\beta$ in one point and $D_{2}$ meets $\beta$ in as many points as $E$ does, say $n$. Then $\chi_{\beta}(E)=n$, but $\chi_{\beta}\left(D_{1} \cup D_{2}\right)=n-1$. This contradicts the $\beta$-tautness of $E$. Then $c$ or $d_{-}$are in $E$. 
3.10. Suppose first $\partial M_{n}$ has only one boundary component. This boundary component has two innermost sutures, which determine two disks, and by 1.9 one is in $R_{+}$and one in $R_{-}$, denoted by $D_{+}$and $D_{-}$respectively. Denote by $E_{-}, E_{+}$the annulus adjacent to $D_{+}$and $D_{-}$respectively. Note that there may be more annuli between $E_{+}$and $E_{-} . D_{+}$and $D_{-}$contain special vertices by 3.2, and $E_{+}$and $E_{-}$also contain special vertices by 3.3 .

\subsection{Claim. $D_{+}\left(D_{-}\right)$does not contain both $a_{+}$and $b\left(d_{-}\right.$and $\left.c\right)$.}

Proof. Suppose $D_{+}$contains $a_{+}$and $b$. It contains no other special vertex by 3.4. $a_{-}$is in $E_{-}$.

Subclaim 1 . No special vertex is in $E_{-}$, other than $a_{-}$.

If $c$ or $d_{-}$are in $E_{-}$then $d_{-}$or $c$ will be the only special vertex in $D_{-}$ and $E_{+}$will contain only $d_{+}$, contradicting 3.6 or 3.9.

Subclaim 2. There is no edge joining the positive side of $b$ and $a_{-}$.

Suppose $\eta$ is such an edge, joining $a_{-}$and $b$ and crossing $\partial D_{+}$. So there is a zero index disk $q$ whose boundary consists of $\eta$ plus an arc over $\beta$ going from $a_{+}$to $b$ (this is the only possibility, for otherwise $I(q)>0$ ). $\eta$ plus the arc joining $a_{+}$and $a_{-}$divide the complement of $D_{+}$in two parts. One of these parts is a disk $E^{\prime}$ contained in $E_{-}$. If $E^{\prime}$ contains simple vertices then there would be more than $p$ edges coming from simple vertices and crossing $\partial D_{+}$, but in $D_{+}$there is room only for $p$ of these edges, so no vertex is in $E^{\prime}$. It is not difficult to see, using $q$ and $Q_{0, n}$, that there is a disk $F \subset M_{n}$, so that $\partial F=\alpha \cup \lambda, \alpha$ is an arc over $D_{+}$joining $a_{+}$and $b, \lambda$ is an arc over $\beta$, going from $a_{+}$to $b$, (int $\left.F\right) \cap \beta=\varnothing$; use $F$ to isotope $D_{+}$and find a surface homologous to $D_{+}$but intersecting $\beta$ in fewer points, contradicting the $\beta$-tautness of $D_{+}$(this is the same argument as in [ $\left.\mathrm{Sc}_{3}, 6.2\right]$, which was applied in 1.15).

Subclaim 3. No edge adjacent to the positive side of $b$ or a simple vertex lying in $D_{+}$crosses $\partial D_{+}$.

If there were one it had to meet the negative side of a special vertex lying in $E_{-}$, but there is no such special vertex.

Subclaim 4. No edge adjacent to $a_{+}$crosses the suture $\partial D_{+}$(other than $\left.\varepsilon_{a}\right)$

If there is one, then by an argument similar to 3.6, Claim 2, there are $p+1$ edges which cross $\partial D_{+}$, at least one of them has to come from the positive side of $b$ or from a simple vertex, which contradicts Subclaim 3.

Then the $p$ edges adjacent to the negative side of $b$ are the only edges which cross $\partial D_{+} . a_{+}$has labels $1,2, \ldots, p$ and is antiparallel to all other vertices in $D_{+}$. Relabel $a_{+}$by $1^{*}, 2^{*}, \ldots, p^{*}$, then now it appears like a vertex parallel to all other vertices in $D_{+}$. An application of 2.8 -where $a_{+}$and the positive side of $b$ are considered the boundary vertex-yields a contradiction.

3.12 Claim. Suppose that $a_{+}$is the only special vertex in $D_{+}$, then we have the following possible cases:

Case 1. $c$ is in $D_{-}, a_{-}$and $d_{-}$are in $E_{-}, b$ and $d_{+}$are in $E_{+}$.

Case 2. $d_{-}$is in $D_{-}, a_{-}$and $c$ are in $E_{-}, d_{+}$and $b$ are in $E_{+}$.

Proof. If $a_{+}$is the only special vertex in $D_{+}$, then by 3.6 there are simple vertices in $D_{+}$and either $c$ or $d_{-}$are in $D_{+}$, but not both of them. If $c$ is in $E_{-}$, then $d_{-}$is in $D_{-}, d_{+}$is in $E_{+}$, and $b$ has to be in $E_{+}$too (by 3.6), so 
we have Case 2 . Note that in this case there may be more annuli between $E_{+}$ and $E_{-}$.

Suppose now $d_{-}$is in $E_{-}$and $c$ is in $D_{-}$. There are $2 p$ edges which cross the suture $\partial D_{-}$.

Subclaim. No edge adjacent to the negative side of $c$ crosses $\partial D_{-}$.

Proof. There are $p$ edges which come from $D_{+}$and meet $d_{-}$, all of them come from simple vertices (3.6, Claim 2). Let $\eta$ be one of these edges. There is an index zero disk $q$ which contains $\eta$ in its boundary, $\partial q$ also contains an arc on $\beta$ joining simple vertices, an arc on $\beta$ going from $d_{-}$to $c$, and an arc joining the negative side of $c$ and a simple vertex, which crosses no suture (this is the only possibility for otherwise $I(q)$ would be positive or there would be a loop in $D_{-}$, contradicting 2.4). This implies that the edges adjacent to the negative part of $c$ meet a simple vertex lying in $D_{-}$, so they do not cross $\partial D_{-}$.

There are $2 p$ edges which cross the suture $\partial D_{-}$, which come from simple vertices or the positive side of $c$. These coming from simple vertices have to meet the negative side of a vertex, so $b$ has to lie in $E_{+}$. Those edges coming from the positive side of $c$, have to meet $d_{+}$or the negative side of a vertex. Meeting $d_{+}$is the only possibility left, so $d_{+}$has to lie in $E_{+}$. Therefore we have Case 1. Note that in this case there are no more annuli in $R(\gamma)$.

Dually we have the following

3.13 Claim. Suppose that $d_{-}$is the only special vertex in $D_{-}$, then we have either Case 2 as in 3.12 or

Case 3. $b$ is in $D_{+}, a_{-}$and $c$ are in $E_{-}, a_{+}$and $d_{+}$are in $E_{+}$.

3.14 Claim. Suppose that $b$ is the only special vertex in $D_{+}$, then one of the following cases occur:

Case 3 as in 3.13 .

Case 4. $a_{-}$and $d_{-}$are in $D_{-}, c$ is in $E_{-}, a_{+}$and $d_{+}$are in $E_{+}$.

Case 5. $a_{-}$and $c$ are in $D_{-}, d_{-}$is in $E_{-}, a_{+}$and $d_{+}$are in $E_{+}$.

Case 6. $c$ is in $D_{-}, a_{-}$and $d_{-}$are in $E_{-}, a_{+}$and $d_{+}$are in $E_{+}$.

Proof. Suppose first that $a_{-}$is in $E_{-}$. Then by 3.9 either $c$ or $d_{-}$are in $E_{-}$. If $c$ is in $E_{-}$, then $d_{-}$is the only special vertex in $D_{-}$, and by $3.6 a_{+}$is in $E_{+}$, so we have Case 3 . If $d_{-}$is in $E_{-}, c$ is in $D_{-}$, and $a_{+}$and $d_{+}$are in the same component, then because there should be special vertices in $E_{+}$it follows that $a_{+}$and $d_{+}$are in $E_{+}$and so we have Case 6.

Now suppose that $a_{-}$is not in $E_{-} \cdot c$ or $d_{-}$are in $E_{-}$but not both, for $a_{-}$would be the only special vertex in $D_{-}$. If $c$ is in $E_{-}$, then $d_{-}$is in $D_{-}$, and by 3.13 no case is possible if $d_{-}$is the only vertex in $D_{-}$, so $a_{-}$is in $D_{-}$ and we have Case 4.

Subclaim. If $d_{-}$is in $E_{-}$then $a_{+}$is in the area adjacent to $E_{-}$.

There are $2 p$ edges crossing $\partial D_{+}, p$ of them come from simple vertices or the positive side of $b$ and have to meet $d_{-}$. The remaining $p$ edges cannot meet a special vertex in $E_{-}$, and so come from the negative side of $b$. Doing an argument similar to 2.6 (recalling $\varepsilon_{d}$ ) shows that for each edge adjacent to the negative side of $b$ we can find a path in $E_{-}$which eventually has to cross 
$\partial E_{-}$and then meet the negative side of a vertex, that is, it has to meet $a_{+}$. So $a_{+}$is in the area adjacent to $E_{-}$.

If $d_{-}$is in $E_{-}$then $c$ is in $D_{-}$, and by the subclaim $a_{+}$and $d_{+}$are in the same component, so $E_{+}$has to be adjacent to $E_{-}$, for otherwise there will not be special vertices in $E_{+}$. Then we have Case 5 .

Dually we have the following

3.15 Claim. Suppose that $c$ is the only special vertex in $D_{-}$, then one of the following cases occur:

Case 1 as in 3.12 .

Case 6 as in 3.14 .

Case 7. $a_{+}$and $d_{+}$are in $D_{+}, b$ is in $E_{+}, a_{-}$and $d_{-}$are in $E_{-}$.

Case 8. $b$ and $d_{+}$are in $D_{+}, a_{+}$is in $E_{+}, a_{-}$and $d_{-}$are in $E_{-}$.

In $3.12,3.13,3.14,3.15$ we have described all the cases in which only one special vertex is in an innermost disk. If an innermost disk contains two of $a_{ \pm}, d_{ \pm}$, then the other innermost disk contains only a single special vertex, so these cases are covered. Therefore the only remaining case is when $b$ and $c$ are in the innermost disks but are not the only special vertex there. By 3.11 it is not possible that $b$ and $a_{+}$are in the same innermost disk, so the only remaining case is the following:

Case 9. $b$ and $d_{+}$are in $D_{+}, a_{-}$and $c$ are in $D_{-}, d_{-}$is in $E_{-}, a_{+}$is in $E_{+}$.

3.16. When $\partial M_{n}$ has only one boundary component the following are all the possible configurations of the vertices. Recall that this boundary component has two innermost sutures, one is in $R_{+}$and one in $R_{-}$, denoted $D_{+}$and $D_{-}$ respectively, and $E_{-}, E_{+}$are the annuli adjacent to $D_{+}$and $D_{-}$respectively.

Case $1 . a_{+}$is in $D_{+}, c$ is in $D_{-}, a_{-}$and $d_{-}$are in $E_{-}, b$ and $d_{+}$are in $E_{+}$.

Case 2. $a_{+}$is in $D_{+}, d_{-}$is in $D_{-}, a_{-}$and $c$ are in $E_{-}, d_{+}$and $b$ are in $E_{+}$.

Case 3. $b$ is in $D_{+}, d_{-}$is in $D_{-}, a_{-}$and $c$ are in $E_{-}, a_{+}$and $d_{+}$are in $E_{+}$.

Case 4. $b$ is in $D_{+}, a_{-}$and $d_{-}$are in $D_{-}, c$ is in $E_{-}, a_{+}$and $d_{+}$are in $E_{+}$.

Case 5. $b$ is in $D_{+}, a_{-}$and $c$ are in $D_{-}, d_{-}$is in $E_{-}, a_{+}$and $d_{+}$are in $E_{+}$.

Case 6. $b$ is in $D_{+}, c$ is in $D_{-}, a_{-}$and $d_{-}$are in $E_{-}, a_{+}$and $d_{+}$are in $E_{+}$.

Case 7. $c$ is in $D_{-}, a_{+}$and $d_{+}$are in $D_{+}, b$ is in $E_{+}, a_{-}$and $d_{-}$are in $E_{-}$.

Case 8. $c$ is in $D_{-}, b$ and $d_{+}$are in $D_{+}, a_{+}$is in $E_{+}, a_{-}$and $d_{-}$are in $E_{-}$.

Case 9. $b$ and $d_{+}$are in $D_{+}, a_{-}$and $c$ are in $D_{-}, d_{-}$is in $E_{-}, a_{+}$is in $E_{+}$.

Note that in Cases 2, 4, 7, 9 there may be more annuli between $E_{-}$and $E_{+}$, but in Cases 1, 3, 5, 6, 8 there are not more annuli. See Figure 9.

Suppose now that $\partial M_{n}$ has more than one boundary component. Indeed, by 3.3 there are at most three, but we will shortly see that it has only two. 

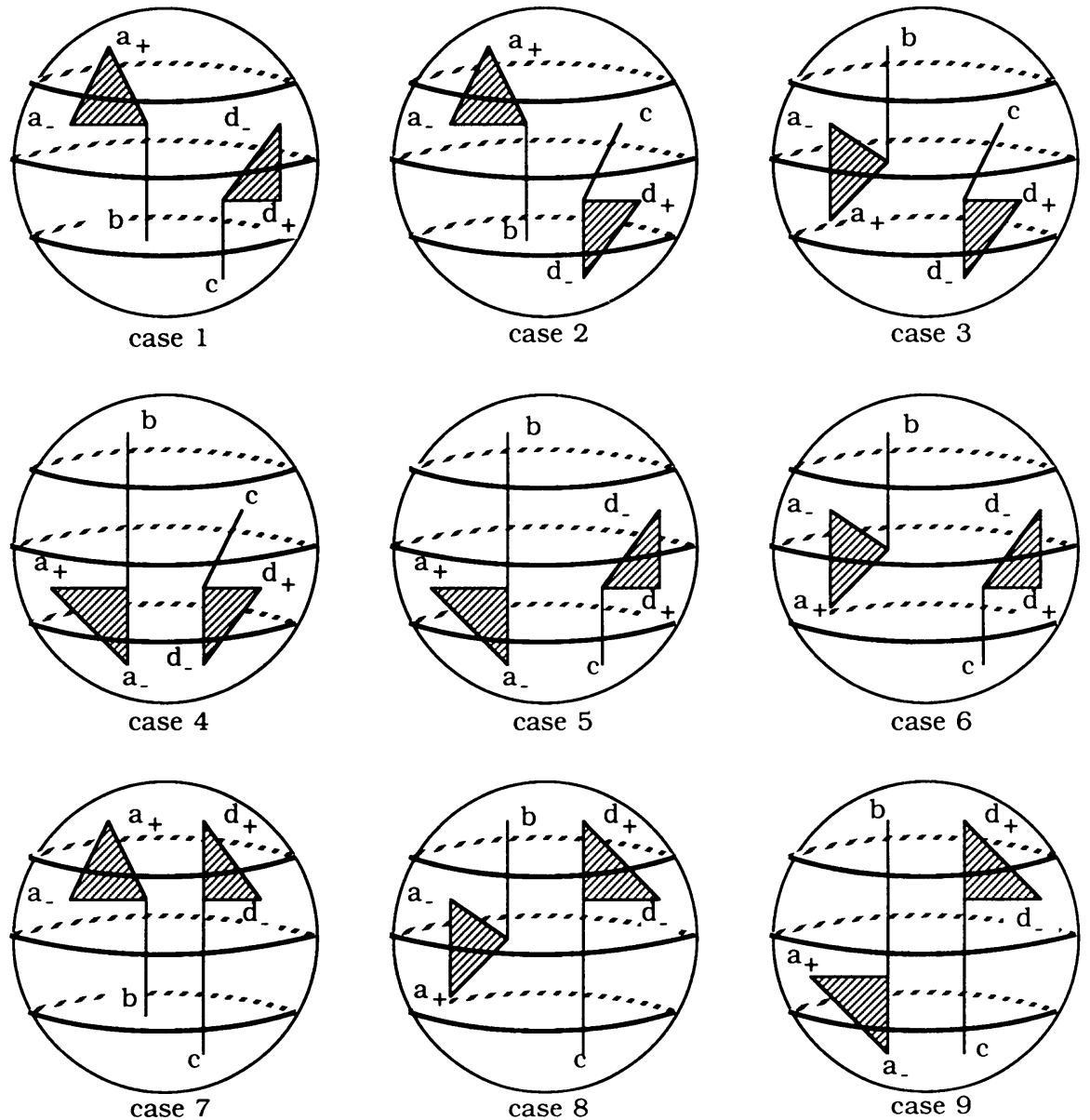

Figure 9

3.17 Claim. A component of $\partial M_{n}$ meets both $A_{+}$and $A_{-}$.

Proof. Suppose a boundary component $T$ meets only $A_{+}$. We have the following cases:

Case 1. $T$ meets only $a_{+}$and $a_{-}$.

Then $T$ has only one suture $\gamma$, and two disks $D_{+}, D_{-}$. If $T$ does not meet simple vertices, then there is a sphere parallel to $T$ meeting $\beta_{\alpha}$ in one point, showing that the band is trivial. If $D_{+}$meets simple vertices, then there are $p$ edges coming from simple vertices which cross $\partial D_{+}$and they have to meet the negative side of a vertex in $D_{-}$, but there is no such vertex. If $D_{-}$ contains simple vertices and $D_{+}$does not, then an argument similar to that in 3.7 (Claim) shows that either there is a Scharlemann cycle in $D_{-}$or a $1^{*}$-path joining $a_{-}$and $a_{+}$, which consists of level edges and bounds, together with $\varepsilon_{a}$, a disk with interior disjoint from $\Gamma$; as in 3.7 this yields a contradiction.

Case 2. $T$ meets only $b$.

This contradicts 3.3 (note that by $1.8 T$ has sutures).

Case 3. $T$ meets $a_{+}, a_{-}$, and $b$.

If $a_{+}$and $b$ are in the same innermost disk, then because $T$ does not meet 

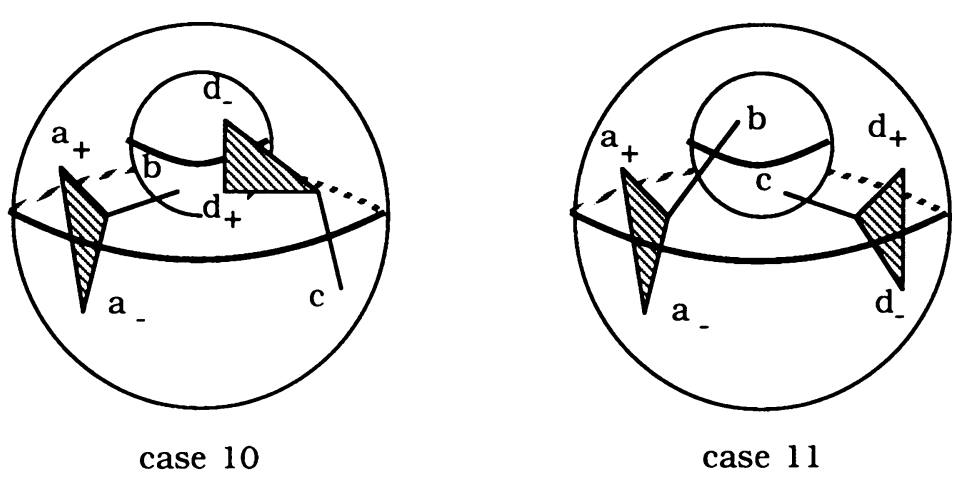

Figure 10

$A_{-}$, an argument similar to 3.11 yields a contradiction. If $b$ is in an innermost disk and $a_{+}$is in the other, then there is an annulus between these disks, which only contains $a_{-}$, which contradicts 3.9. If $b$ is an innermost disk and $a_{-}$is in the other, then there would not be special vertices in the area adjacent to $b$, which contradicts 3.3.

3.18. Note that 3.17 implies that $\partial M_{n}$ has only two components, denote them by $T$ and $T^{\prime}$. Applying 3.17 it is not difficult to see that we have only the following two cases. See Figure 10.

Case 10. $T$ meets $a_{+}, a_{-}$and $c$, and $T^{\prime}$ meets $d_{+}, d_{-}$and $b$. By 3.6 and 3.7 each sphere contains only one suture; $a_{-}$and $c$ are contained in a disk of $T$, and $a_{+}$in the other; $d_{+}$and $b$ are in a disk of $T^{\prime}$, and $d_{-}$in the other.

Case 11. $T$ meets $a_{+}, a_{-}, d_{+}, d_{-}$and $T^{\prime}$ meets $b$ and $c$. By 3.3, 3.6 and 3.7 each sphere contains only one suture; a disk of $T$ contains $a_{+}$and $d_{+}$, and the other contains $a_{-}$and $d_{-}$; a disk of $T^{\prime}$ contains $b$ and $c$ is in the other.

\section{Finding a SET OF PARALlel EDgeS}

We will concentrate on Case 4 of 3.16, because it is representative, and the proofs for the remaining cases are similar.

4.1 Claim. If Case 4 of 3.16 happens then the orientation of $\beta$ is as in the Figure 3(a).

Proof. 2.5 implies that there are at least $2 p$ edges crossing the suture $\partial D_{+} \cdot p$ of them come from the positive side of $b$ or from simple vertices and have to meet $c$ in its negative side, for in the negative side of $c$ there is room only for $p$ edges. Then $2 p$ edges across $\partial D_{+}, p$ comes from simple vertices or the positive side of $b$, and $p$ from the negative side of $b$. This implies that no edge in $D_{-}$crosses $\partial D_{-}$(other than $\left.\varepsilon_{a}, \varepsilon_{d}\right)$, for otherwise there would be a component of $Q_{n}$ with positive index. If $\beta$ has the orientation of Figure 3(b), $d_{-}$has labels $1,2, \ldots, p . d_{-}$is antiparallel to all vertices in $D_{-}$. Relabel $d_{-}$by $1^{*}, 2^{*}, \ldots, p^{*}$, so that it comports like a vertex parallel to all vertices in $D_{\text {- }}$. Note that the parity rule still holds under the new labelling. Apply 2.8 to this situation, considering $a_{-}$and $d_{-}$as the vertex in the boundary. So 2.8 implies that there is a Scharlemann cycle in $D_{-}$, which is a contradiction. 
Then the orientation of $\beta$ is as in Figure 3(a), and the negative side of $c$ is in the bottom of $\beta$.

4.2 Claim. All the edges adjacent to the positive side of $b$, the negative side of $c, a_{-}$and $d_{-}$are level.

Proof. As in the proof of $4.1,2 p$ edges cross $\partial D_{+}, p$ come from simple vertices or the positive side of $b$ and meet the negative side of $c$, and $p$ come from the negative side of $b$. So no edge crosses $\partial D_{-}$(other than $\left.\varepsilon_{a}, \varepsilon_{d}\right)$. As $\beta$ has the orientation of Figure 3(a), $d_{-}$has labels $1^{*}, 2^{*}, \ldots, p^{*}$. Relabel $d_{-}$by $1,2, \ldots, p$, so that it comports like a vertex parallel to all the vertices in $D_{-}$. Apply 2.7 to this situation, considering $a_{-}$and $d_{-}$as the vertex in the boundary. Then 2.7 shows that the edges adjacent to $a_{-}$and $d_{-}$are level. This implies that the edges adjacent to the negative side of $c$ and to the positive side of $b$ are level.

4.3. $I(Q)=I\left(Q_{n}\right)=0$, so by 1.17 we can assume that $Q_{1}=Q-$ int $\eta(S)$, $Q_{i}=Q_{i-1}-$ int $\eta\left(S_{i}\right)$, for all $Q_{i}$ in the hierarchy, and then $\partial Q_{n}=\left(Q \cap \partial M_{n}\right) \cup$ $\left(Q \cap \beta_{n}\right)$. Also $Q \cap T=\partial Q_{n} \cap T$.

Look at the graph $\Lambda$ in the planar surface $Q_{\alpha}$ formed by its intersection with the sphere $T \subset \partial M_{n}$. A vertex of $\Lambda$ is a circle of intersection between $\beta$ and $Q_{\alpha}$, and the edges are the arcs of intersection between $Q_{\alpha}$ and $T$. As in 2.1 denote the vertices of $Q_{\alpha}$ by $a_{1}, a_{2}, \ldots, a_{p}$. We say that a loop $\eta$ in $\Lambda$ is bad if the two circles of intersection of $Q_{\alpha}$ with $\partial M$ are in distinct components of $Q_{\alpha}-\eta$, otherwise it is good. Note that a level edge in $\Gamma$ correspond to a loop in $\Lambda$. Choose one of the boundary components of $Q_{\alpha}$ which lie in $\partial M$, denote it by $Y$; define the interior of a loop in $Q_{\alpha}$ as the component of its complement which does not contain $Y$. Label the vertices of $T$ with $1,2, \ldots, n ; 1$ corresponds to $a_{ \pm}, 2$ to $b, n-1$ to $c$, and $n$ to $d_{ \pm}$, label the other vertices in the obvious way. Label the end of an edge in $a_{i}$ with $j$ $\left(j^{*}\right)$ if this point corresponds to an intersection between $a_{i}$ and the vertex in $T$ labeled with $j$, and it lies in the top (bottom) of $\beta$.

4.4 Claim. The level edges adjacent to the positive side of $b$, the negative side of $c, a_{-}$, and $d_{-}$are bad loops in $\Lambda$.

Proof. We distinguish two cases.

Case 1. There are arcs of $\beta$ running from $D_{+}$to $D_{-}$. So there are more vertices in $\Gamma$.

In this case there are $2 p$ edges crossing $\partial D_{+}, p$ coming from the negative side of $b$ and $p$ coming from simple vertices.

Step 1 . The level edges adjacent to the positive side of $b$ correspond to bad loops in $\Lambda$.

If one of them is a good loop, then there is an innermost one; let $\eta$ be this loop. $\eta$ in $\Gamma$ has an end in the positive side of $b$ and the other end in the top part of a simple vertex, its ends in $\Gamma$ are labeled with $i^{*}-i$, say. $\eta$ in $\Lambda$ is a loop based at $a_{i} . \eta$ in $\Lambda$ looks as in Figure 11. Now because $\eta$ is not adjacent to $a_{ \pm}, d_{ \pm}$, the Seifert surface $S$ has to meet $Q_{\alpha}$ in an arc $\sigma$ as shown in Figure 11, $\sigma$ has one end in $\beta_{0}$, say, and the other in $\beta_{\alpha}$. If the other end of $\sigma$ is in the bottom part of $\beta$, this would contradict that the orientation of $\beta$ points in the direction of the normal vector to $S$; if this end of $\sigma$ is in the top part of $\beta$, then $\sigma$ will cut a disk $q_{1}$, with $I\left(q_{1}\right)=-2$, which will be 


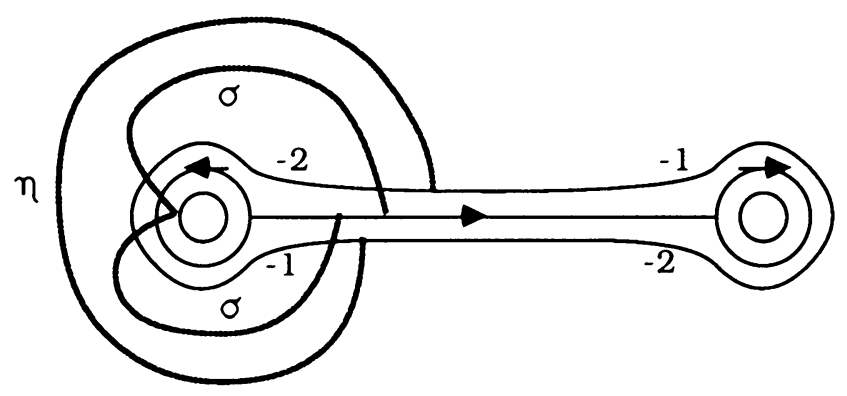

FIGURE 11

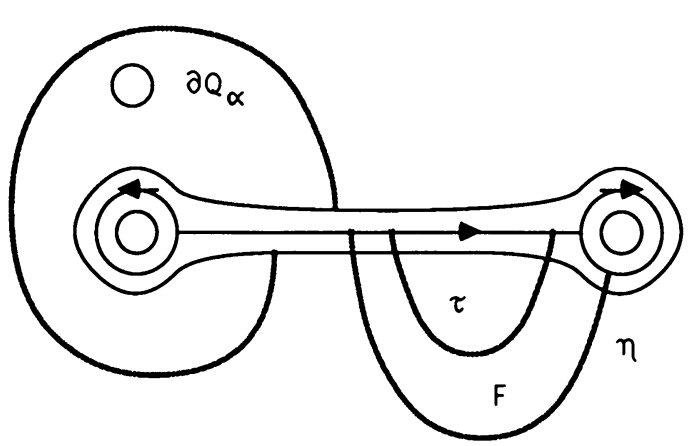

FIGURE 12

a parameterizing surface in its own right in $M_{1}$, and then $I\left(q_{n}\right) \leq-2$, which contradicts 1.15 (an alternate argument: Note that in this situation there is an isotopy which moves the Seifert surface intersecting $\beta$ in fewer points, which contradicts the $\beta$-tautness of $S$ ).

Note that the loops corresponding to the level edges in $a_{-}$are parallel to these corresponding to $b$, so they have to be bad loops (this is because there is a disk $q$ of $Q_{n}$ which made them parallel).

Step 2. The level edges ending in the negative side of $c$ are bad loops in $\Lambda$.

Each of these edges join $c$ and a simple vertex, with both ends in the bottom part of these vertices. Suppose one of these edges corresponds to a good loop $\tau$ in $\Lambda$; let $\eta$ be the loop parallel to $\tau$ which corresponds to a level edge adjacent to $d_{-}$, so $\eta$ is a good loop. We have something similar in Figure 12. Let $F$ be the disk in $Q_{\alpha}$ bounded by $\eta . \tau$ is inside $F$ and there are no vertices inside $F$ because each vertex in $\Lambda$ has a bad loop by Step 1 . Note that $\eta$ in $T$ crosses no suture. Let $S_{i}$ be the first surface in the hierarchy which meets the interior of $F$. Then either $S_{i}$ meets $F$ in a closed curve, which will form a disk of $Q_{i}$ with boundary contained in $R(\gamma)$, contradicting that $Q_{i}$ is a parameterizing surface, or $S_{i}$ meets $F$ in a collection of arcs, each with ends in the bottom part of $\beta_{\alpha}$. But the two intersections of $\beta$ and $S_{i}$ corresponding to the ends of one of these arcs have a different sign, which contradicts the $\beta$-tautness of $S_{i}$. This implies that the loops in $\Lambda$ corresponding to the level edges adjacent to the negative side of $c$ and $d_{-}$are bad loops.

Case 2. There is no simple vertex in $D_{+}, D_{-}$.

Then the positive side of $b$ and the negative of $c$ are joined up by $p$ level edges, and so are $a_{-}$and $d_{-}$. If one of these level edges corresponds to a good 


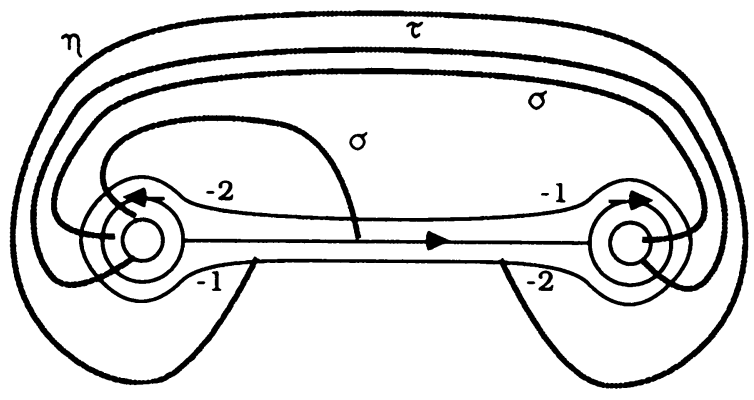

FIGURE 13

loop in $\Lambda$, then there is an innermost one. Let $\eta$ be a level edge joining $b$ and $c$ which corresponds to an innermost good loop in $\Lambda$; let $\tau$ be the parallel loop corresponding to an edge joining $a_{-}$and $d_{-}$, and note that $\tau$ crosses no suture. If $\eta$ is inside $\tau$ then proceed as in Step 2 above. So suppose $\tau$ is inside $\eta$, we have something similar in Figure 13. Look how the surface $S$ meets $Q_{\alpha}$ in the vertex in question; it meets $Q_{\alpha}$ in one arc $\sigma$, with one end in $\beta_{0}$, and the other end either in $\beta_{\alpha}$ or $\beta_{1}$. In any case $S$ cut a disk $q_{1}$ with $I\left(q_{1}\right)=-2$ which is a parameterizing surface in its own right in $M_{1}$, therefore $q_{n}$ has negative index, which contradicts 1.15 .

4.5 Remark. If $p>1$, there are two possibilities of how the negative side of $c$ joins the vertices of $D_{+}$. Let $\eta_{1} \quad\left(\eta_{p}\right)=$ (edge adjacent to $c$ at label $1^{*}$ $\left.\left(p^{*}\right)\right) \cap E_{-}$. Let $\alpha$ be the arc in $c$, which go through the negative side of $c$, and which joins the labels $1^{*}$ and $p^{*} . \eta_{1} \cup \eta_{p} \cup \alpha \cup\left(\operatorname{arc}\right.$ in $\partial D_{+}$joining $\eta_{1}$ and $\eta_{p}$ ) bounds a disk $F \subset E_{-}$. If the positive side of $c$ is in the interior of $F$, so are the edges coming from the negative side of $b$. Doing an argument as in 4.2 we can show that all the edges adjacent to the positive side of $c$ and negative side of $b$ are level, and doing an argument as in 4.4 they correspond to bad loops in $Q_{\alpha}$. In this case 4.6 and 4.7 are easier. If the positive side of $c$ is not in $F$, all the edges adjacent to the negative side of $c$ lie in $F$, and no other vertex or edge is in $F$. In this case we cannot assure that the edges adjacent to the negative side of $b$ are level. This made the complications of 4.6. Also an edge adjacent to the negative side of $b$ may cross two sutures and meet $a_{+}$. An edge adjacent to the positive side of $c$ may cross a suture and meet $d_{+}$.

4.6 Claim. Suppose that there are simple vertices in $D_{+}, D_{-}$. Then there is a vertex $v=a_{x}$ in $Q_{\alpha}$ so that all the edges adjacent to $v$ are loops, except possibly those with labels $1,2,3,4\left(\right.$ or $\left.(n-2)^{*},(n-3)^{*}\right)$, and all the loops are bad loops, except possibly one with labels $n, n-1$ or 1,2 , which correspond in $\Gamma$ to an edge joining the positive side of $c$ and $d_{+}$, or an edge joining the negative side of $b$ and $a_{+}$.

Proof. 4.4 says that in $\Lambda$ each vertex has bad loops with one end labeled $1^{*}, 2^{*},(n-1)^{*}, n^{*}$. Take an innermost vertex $v=a_{x}$ in $\Lambda$, so in one of the sides determined by the loops at $v$ there are no more vertices. Let $\eta_{i}$ be the loop at $v$ with one label $i^{*}$, for $i=1,2, n-1, n$. Then either $\eta_{1}$ of $\eta_{n-1}$ contains the other three loops in its interior, suppose first that $\eta_{1}$ is the outermost of these loops. The other end of $\eta_{1}\left(\eta_{2}\right)$ is labeled $j(j+1), j<n-2$. 
The other end of $\eta_{n-1}\left(\eta_{n}\right)$ is labeled $(k+1)^{*}\left(k^{*}\right)$. If $k+2 \neq n-1$ then there is a loop at $v$ with labels $s^{*}, t^{*}, k+2<s<t<n-1$ but this implies that in $T$ there are simple vertices joined by an edge with labels $x^{*}-x^{*}$; this edge cannot cross a suture, for no edge joining simple vertices crosses a suture, so the two vertices are parallel, which contradicts the parity rule. So $k+2=n-1$, and $\eta_{n-1}$ is an innermost loop.

Suppose first that there is a good loop in $v$, with labels $n, n-1$ (note that this is the only possible good loop in the interior of $\eta_{1}$, any other good loop would contradict the parity rule, as above; this loop corresponds to a level edge with labels $x$ joining $c$ and $\left.d_{+}\right)$. So because $k+2=n-1$ the number of labels between the labels $2^{*}$ and $k^{*}$, which is $n-6$, has to be equal to the number of labels between $j+1$ and $n-1$, and this forces that $j=3$, i.e. all the edges incident to $v$ are loops, except perhaps those incident to the labels 1,2 .

Suppose now that there is no good loop at $v$. Then incident to the labels $n$, $n-1$ are two bad loops, for these labels are the interior of $\eta_{1}$. The number of labels between the labels $2^{*}$ and $k^{*}$, which is $n-6$, has to be equal to the number of labels between $j+1$ and $n^{*}$, and this forces that $j=5$, and then all the edges incident to $v$ are loops, except perhaps the edges with labels $1,2,3,4$.

If $\eta_{n-1}$ is the outermost loop then a similar argument shows that all the edges adjacent to $v$ are loops except perhaps those with labels $(n-2)^{*},(n-3)^{*}$. The only possible good loops have ends labeled 1,2 or $n, n-1$. This completes the proof.

4.7 Claim. In $\Gamma$ there are two vertices joined by $p$ parallel level edges, one for each label, which cross no suture, and these edges correspond to bad loops in $\Lambda$.

Proof. Suppose first that there are simple vertices in $D_{+}, D_{-}$. Note that in $T$ two vertices labelled $i, i+1$ are antiparallel, so only one of 3,4 (or $n-2, n-3)$ can be at $D_{+} .4 .6$ implies that there is a label $x \in\{1, \ldots, p\}$, so that all the edges in $D_{+}$incident to a label $x$ or to a label $x^{*}$ have to be level, except possibly one, i.e. the incident to the vertex $3,4, n-2$ or $n-3$. But in $D_{+}$ there is the same number of labels $x$ and $x^{*}$, and there is exactly one edge with label $x$ (the adjacent to $b$ ) and one with label $x^{*}$ (joining a simple vertex and $c)$ in $E_{+}$which cross $\partial D_{+}$, which forces that all the edges adjacent to a label $x^{*}$ or $x$ are level. Take a $x^{*}$-path $\gamma$ in $D_{+}$beginning at $b$. It is formed by level edges and ends at $c$, but it must meet all the vertices in $D_{+}$, for otherwise there would be a $x^{*}$-cycle formed with the vertices missed by the path. Say $\gamma$ meets the vertices in the order $v_{0}=b, v_{1}, v_{2}, \ldots, v_{m}=c . \gamma \cup$ (edge incident to $b$ at label $x$ ) separates $D_{+}$in two parts. Take now an $(x-1)^{*}$-path $\lambda$ (or a $\left.(x+1)^{*}\right)$ beginning at $b$; all its edges lie in one side of $\gamma . \lambda$ eventually crosses $\partial D_{+}$and meets the negative side of $c$. If $\lambda$ does not meet the vertices in $D_{+}$ in the same order as $\gamma$, then there is a first vertex at which they disagree, say $\lambda$ meets the vertices in the order $v_{0}, v_{1}, \ldots, v_{k}, v_{s}, s \neq k+1$. If $s<k$, then $\lambda$ is a $i^{*}$-cycle; if $s>k$, then the part of $\gamma$ from $v_{k}$ to $v_{s}$ and the edge of $\lambda$ joining $v_{k}$ and $v_{s}$ enclose an area which has $P(x-1)$, so there would be an $(x-1)$-cycle, and then a Scharlemann cycle (cf. Lemma 2.3). Then $\lambda$ meets the vertices in the same order as $\gamma$ does, so it consists of level edges. Repeat 
the same process for $(x-2)^{*}$, etc. This implies that all the edges in $D_{+}$are level and that two consecutive vertices in the path $\gamma$ are joined by $p$ parallel level edges. In particular this shows that the positive side of $b$ is joined to a simple vertex by $p$ level edges. By 4.4 , Case 1 , these edges correspond to bad loops in $\Lambda$.

If there is no simple vertex in $D_{+}, D_{-}$, then $a_{-}$and $d_{-}$are joined by $p$ level edges which cross no suture, and by 4.4 , Case 2 , these edges correspond to bad loops in $\Lambda$.

4.8. Now we outline a proof of 4.6 for the remaining cases. Cases 5, 7, 8 are similar to Case 4 . In these cases $b$ or $c$ is the only special vertex in $D_{+}$, there are $2 p$ edges which cross the suture $\partial D_{ \pm}, p$ come from the negative side of $b$ or $c, p$ come from simple vertices or the positive side of $b$ or $c$ and meet a special vertex, and in $E_{\mp}$ there is only one special vertex. An argument similar to 4.1 shows that Case 7 is possible only when $\beta$ is oriented as in Figure 3(a), and Cases 5, 8 are possible only when $\beta$ is oriented as in Figure 3(b). Now we proceed with $4.2,4.4,4.6,4.7$, as before, interchanging the roles of $b$ and $c$ when necessary.

In cases $1,2,3, a_{+}$or $d_{-}$is the only special vertex in $D_{ \pm}$, so 3.6 implies that $D_{ \pm}$has simple vertices and no edge adjacent to $a_{+}$or $d_{-}$crosses the suture $\partial D_{ \pm}$, so there are $p$ edges which cross $\partial D_{ \pm}$, all coming from simple vertices and have to meet a special vertex in $E_{ \pm}$. This implies that in Case 1(3) there are $2 p$ edges which cross $\partial D_{-}\left(\partial D_{+}\right), p$ come from simple vertices and meet the negative side of $b(c), p$ come from the positive side of $c(b)$ and meet $d_{+}\left(a_{-}\right)$. Also in Case 1(3) the $p$ edges adjacent to the positive side of $b \quad(c)$ have to cross a suture and join $a_{-}\left(d_{+}\right)$. An argument as in 4.1 shows that if $\beta$ is oriented as in Figure 3(a) then we only have Case 2; and if $\beta$ is oriented as in Figure 3(b) we have Cases 1, 3. Now proceed with 4.2, 4.4, 4.6, 4.7 as before.

Now consider Case 6 . If there are no simple vertices in $D_{+}\left(D_{-}\right)$, then the $2 p$ edges adjacent to $b(c)$ cross $\partial D_{+}(\partial D)$; the $p$ edges coming from the positive side of $b(c)$ meet $a_{-}\left(d_{+}\right)$, by index restrictions. Then there is a disk $D$, so that $\partial D=\alpha \cup \lambda, \alpha$ is an arc in $\partial T$ joining $b$ and $a_{-}$and $\lambda$ is an arc in $\eta(\beta)$ going from $b$ to $a_{-}$. An argument similar to 3.6, Claim 1, shows that $E_{-}$is not $\beta$-taut, which is a contradiction. Therefore there are simple vertices in $D_{+}\left(D_{-}\right)$. There are $2 p$ edges which cross $\partial D_{+}\left(\partial D_{-}\right)$. If more than $p$ of these edges come from $b(c)$, then an argument similar to 3.6, Claim 2, shows that there are more than $p$ edges coming from simple vertices which cross the suture $\partial D_{+}\left(\partial D_{-}\right)$, but in $d_{-}\left(a_{+}\right)$there is only room for $p$ of them. Then $p$ edges come from simple vertices and meet $d_{-}\left(a_{+}\right)$, and $p$ come from $b(c)$. If an edge $\alpha$ joins a simple vertex in $D_{+}\left(D_{-}\right)$and $d_{-}\left(a_{+}\right)$, then $\alpha$ is part of $a$ index zero disk $q$, whose boundary consist of $\alpha$, an arc on the negative side of $A_{-}\left(A_{+}\right)$, an edge in $D_{-}\left(D_{+}\right)$joining the negative side of $c(b)$ and a simple vertex and which crosses no suture, and another arc on $\eta(\beta)$. This implies that the $p$ edges adjacent to the negative side of $c(b)$ do not cross the suture $\partial D_{-}\left(\partial D_{-}\right)$. Hence the $p$ edges adjacent to the positive side of $b(c)$ cross $\partial D_{+}\left(\partial D_{-}\right)$, and they have to meet $a_{-}\left(d_{+}\right)$. An argument similar to 4.1 shows that Case 6 is possible only when $\beta$ is oriented as in Figure 3(a). Now we follow with $4.2,4.4,4.6,4.7$. 
In Case 9 , if an edge adjacent to $d_{+}\left(a_{-}\right)$crosses $\partial D_{+}\left(\partial D_{-}\right)$, then it meets $d_{-}\left(a_{+}\right)$, which implies that there is a loop at $c(b)$, so no edge adjacent to $d_{+}\left(a_{-}\right)$crosses $\partial D_{+}\left(\partial D_{-}\right)$. Take an $i$-path or $i^{*}$-path $\lambda_{i}$ starting at $d_{+}\left(a_{-}\right)$for each $i$. This path has to cross $\partial D_{+}\left(\partial D_{-}\right)$, and if the edge crossing $\partial D_{+}\left(\partial D_{-}\right)$is incident in $D_{+}\left(D_{-}\right)$to a simple vertex or the positive side of $b(c)$, then that path will finish at $d_{-}\left(a_{+}\right)$. Doing an argument as in 3.7 (Claim), $\lambda_{1}$ or $\lambda_{p}$ starts at $d_{+}$and finishes at $d_{-}$, and consists of level edges. $\lambda_{1} \cap \varepsilon_{d}$ bounds a disk $D$ with interior disjoint from $\Gamma$, as in 3.7 this is not possible. Then the edge of the path $\lambda_{i}$ crossing $\partial D_{+}\left(\partial D_{-}\right)$is incident to the negative side of $b(c)$. Then the $p$ edges adjacent to the negative side of $b(c)$ cross $\partial D_{+}\left(\partial D_{-}\right)$. In particular this implies that no edge adjacent to the positive side of $b(c)$ crosses $\partial D_{+}\left(\partial D_{-}\right)$. This also implies that no edge adjacent to a simple vertex in $D_{+}\left(D_{-}\right)$can cross $\partial D_{+}\left(\partial D_{-}\right)$, by index restrictions. Then the only edges which cross $\partial D_{+}\left(\partial D_{-}\right)$are those adjacent to the negative side of $b(c)$. Doing something like in 4.1 it can be seen that this case is only possible when $\beta$ is oriented as in Figure 3(a). Now follow with 4.2, 4.4, 4.6, 4.7 as before.

Case 10 is similar to Case 9 , and Case 11 is similar to Case 4. These cases are only possible when $\beta$ is oriented as in Figure 3(a).

\section{CONSTRUCTING A SWALlOW-FOllow tORUS}

Recall that $P$ denotes the decomposing sphere from which $Q_{\alpha}$ was obtained. It was proved in 4.7 that there are two vertices in $T\left(=\partial M_{n}\right)$ which are joined by $p$ consecutive parallel level edges, one for each label $i, i=1, \ldots, p$, and these edges correspond to bad loops in $Q_{\alpha}$. Call these vertices $v_{1}$ and $v_{2}$. In what follows we do not distinguish between the labels $i$ and $i^{*}$.

Denote by $e_{i}$ the edge joining $v_{1}$ and $v_{2}$ with label $i$, for $i=1, \ldots, p$; and denote by $\alpha_{i}\left(\eta_{i}\right)$ the arc inn $v_{1}\left(v_{2}\right)$ joining the labels $i$ and $i+1$, for $i=1, \ldots, p-1$. Let $C_{i}$ be the disk in $T$ bounded by $\alpha_{i}, \eta_{i}, e_{i}$, and $e_{i+1}$. The arc $e_{i}$ in $Q_{\alpha}$ (or in $P$ ) is a loop based at $a_{i}$. Choose for each $a_{i}$ an arc $\gamma_{i}$ in $a_{i}$ having the same ends as $e_{i}$, and so that $\alpha_{i}, \gamma_{i}, \eta_{i}, \gamma_{i+1}$ bound a disk in $\partial \eta(\beta)$. Let $H_{i}$ be the disk in $\partial \eta(\beta)$ bounded by $\alpha_{i}, \gamma_{i}, \eta_{i}, \gamma_{i+1}$. Then the interior of $H_{i}$ and $C_{i}$ do not meet $P$. Let $A_{i}$ be the annulus obtained by gluing $C_{i}$ and $H_{i}$ along $\alpha_{i} \cup \eta_{i}$; the interior of $A_{i}$ meets neither $P$ nor int $\eta(\beta)$. Let $F_{i}$ be the annulus in $P$ bounded by $e_{i}, \gamma_{i}, e_{i+1}, \gamma_{i+1}$; note that this annulus may intersect $\eta(\beta)$. It follows that $\partial A_{i}=\partial F_{i}$. See Figure 14. Note that there are two choices for $\gamma_{i}$, which produce two choices for $H_{i}, A_{i}$ and $F_{i}$; denote by $\gamma_{i}^{\prime}, H_{i}^{\prime}, A_{i}^{\prime}, F_{i}^{\prime}$ the alternative choice.

Note that $A_{i}$ and $A_{i+1}$ have a component of its boundary in common, so $A_{i}$ and $A_{i+1}$ lie in different side of $P$ in $S^{3}$. It is not difficult to see that there is a $F_{j}$ which is innermost, i.e. its interior misses $\eta(\beta)$ or meets it in two disks, and in the last case the interior of $F_{j}^{\prime}$ misses $\eta(\beta)$. Do 2-surgery in $P$ with $A_{j}$; then we obtain a sphere $P^{\prime}$ intersecting $k_{b}$ in two points and a torus $R$ disjoint from $k_{b} . P^{\prime}$ and $R$ are isotopic to $\left(P-F_{j}\right) \cup A_{j}, F_{j} \cup A_{j}$, respectively. If $P^{\prime}$ is a decomposing sphere for $k_{b}$, and $F_{j}$ meets $\eta(\beta)$ in two disks, then $P^{\prime}$ has fewer intersections with $\eta(\beta)$ than $P$ does, contradicting the minimality of the intersections between $P$ and $\eta(\beta)$; if the interior of $F_{j}$ misses $\eta(\beta)$, then using $A_{j}^{\prime}$ instead of $A_{j}$, we get a decomposing sphere $P^{\prime \prime}$ 

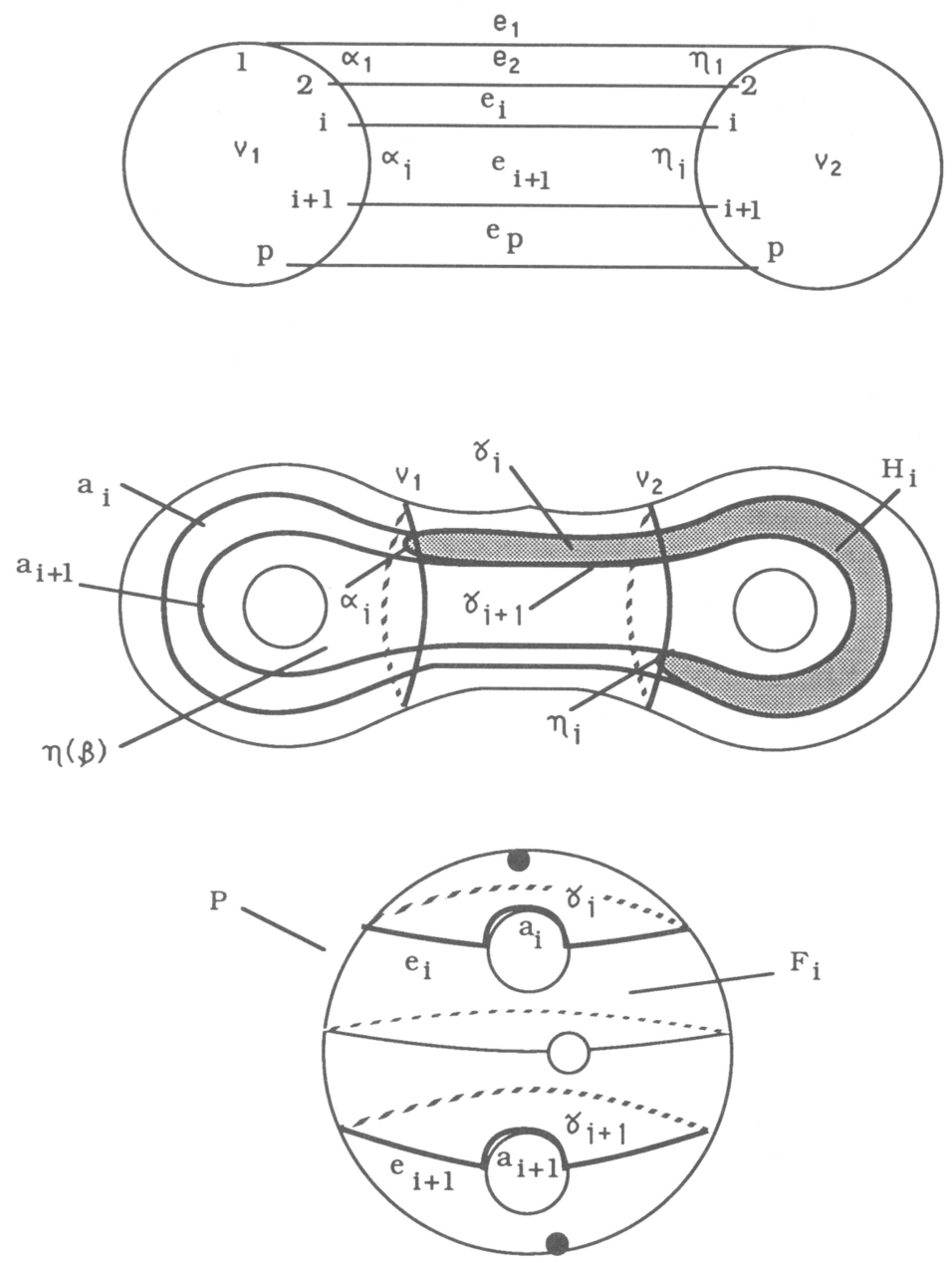

FIGURE 14

(in fact isotopic to $P^{\prime}$ ), having fewer intersections with $\eta(\beta)$ than $P$ does. So $P^{\prime}$ is not a decomposing sphere and the annulus $A_{i}$ runs parallel to one arc of one of the summands of $k_{b}$. Then $R$ is a swallow-follow torus for $k_{b}$. If the interior of $F_{j}$ misses $\eta(\beta)$ then clearly $R$ is disjoint from $\eta(\beta)$. If $F_{j}$ meets $\eta(\beta)$ in two disks, then using $A_{j}^{\prime}$ instead of $A_{j}$, we get a swallow-follow torus $R^{\prime}$ (isotopic to $R$ ) which is disjoint from $\eta(\beta)$.

This construction is possible only if $p>1$; so we conclude that either $p=1$, or there is a swallow-follow torus disjoint from the band.

This completes the proof of Theorem 1.

\section{BAND SUMS OF SPLIT LINKS WHICH YIELD COMPOSITE KNOTS}

Theorem 2. Let $k_{b}$ be a band sum of $k_{0}$ and $k_{1}$, for $k_{0} \cup k_{1}$ a split link. If $k_{b}$ is composite, then there is a decomposing sphere disjoint from the band or there is a decomposing sphere crossing the band in one arc parallel to $b(\{1 / 2\} \times I)$. 
Proof. Clearly $S^{3}-\left(k_{0} \cup k_{1} \cup b(I \times I)\right)$ is irreducible. Now apply Theorem 1 , as stated in the introduction. (d) of Theorem 1 cannot happen because $S^{3}-\left(k_{0} \cup k_{1}\right)$ is reducible. If (a) or (b) happen then we are finished, so suppose (c) happens. Let $T$ be a swallow-follow torus disjoint from the band. $T$ is a torus in the complement of $k_{0} \cup k_{1}$. T bounds a solid torus $T^{\prime}, k_{b}$ lies in $T^{\prime}$. The winding number of $k$ in $T^{\prime}$ is 1 , so the algebraic winding number of $k_{0} \cup k_{1}$ is also 1; so at least one of the components of this link is not trivial inside $T$.

Let $S$ be a splitting sphere for $k_{0} \cup k_{1}$. Recall that $b: I \times I \rightarrow S^{3}$ is a map such that $b^{-1}\left\{k_{i}\right\}=I \times\{i\}, i=0,1$. Isotope $S$ so that intersects the band in a collection of arcs parallel to $I \times\{1 / 2\}$; take a splitting sphere $S$ which minimizes this number of arcs.

Claim. $T$ and $S$ can be chosen so that they do not intersect.

Proof of claim. Suppose they intersect in a collection of circles, take one innermost circle $\alpha$ in $S$, which is boundary of a disk $D$. If $D$ lies outside of $T^{\prime}$, then because $T$ is an essential torus, $\alpha$ is a trivial curve on $T$, which bounds a disk in $T$, and because $S^{3}-T^{\prime}$ is irreducible we can isotope $D$ to remove the circle $\alpha$. If $D$ lies inside $T^{\prime}$, then because the winding number of $k_{0} \cup k_{1}$ in $T^{\prime}$ is $1, D$ cannot be a meridian of $T^{\prime}$, so $\alpha$ is trivial in $T$ and is boundary of a disk $D^{\prime} \subset T$. Take a curve of intersection between $S$ and $D^{\prime}$ which is innermost on $D^{\prime}$; such a curve is a boundary of a disk $D^{\prime \prime} \subset D^{\prime}$ whose interior is disjoint from $S$. By doing surgery in $S$ with $D^{\prime \prime}$, we get two spheres, at least one of them is a splitting sphere which has fewer intersections with $T$, and it has equal or fewer intersections with the band than $S$.

As $T$ is a swallow-follow torus for $k_{b}$, there is a meridian disk of $T^{\prime}$ which intersects $k_{b}$ in one point, call it $Q$. By isotoping $Q$, suppose that it meets the band in a collection of arcs parallel to $b(\{1 / 2\} \times I)$; choose $Q$ so that it minimizes this number of arcs. Now follow the combinatorial route of [BE], the situation in here is very similar to the situation in there, because the disk $Q$, which intersects the knot in one point is like a decomposing sphere for a knot; note also that $S$ does not meet $\partial Q$. So we consider graphs $\Gamma_{S}$ and $\Gamma_{Q}$, in $S$ and $Q$ respectively, whose fat vertices are the disks of intersection between a regular neighborhood of $b(I \times I)$ and $S$ and $Q$ respectively, and their edges are the intersection arcs between $S-\operatorname{int} \eta(b(I \times I)$ and $Q-$ int $\eta(b(I \times I)$. The whole combinatorial argument is now similar to $[\mathrm{BE}]$. The strategy of the proof is as follows: Use [BE, $\S 4]$ (which is essentially 2.3 , i.e. [CGLS, 2.6.2]) to find a pure level circuit or a (Scharlemann) cycle $\lambda$ in $\Gamma_{S}$. If $\lambda$ is a pure level circuit in $\Gamma_{S}$, then it corresponds in $\Gamma_{Q}$ to a collection of loops; if one of these loops is good then we do a path threading argument, as in [ $\left.\mathrm{Sc}_{1}, \S 6,7, \mathrm{BE}, 2.8\right]$. If all these loops are bad, then in [BE, $\S 3] \lambda$ is used to construct two spheres which meet a composite knot $k$ in two points, and one of them had to be a decomposing sphere unless $k$ had a two-bridge knot summand. Here the same construction yields a sphere intersecting $k_{b}$ in two points, and a disk with the same boundary as $Q$, intersecting $k_{b}$ in one point, so it is a meridian disk of $T^{\prime}$. This new disk has fewer intersections with the band than $Q$, contradicting its minimality. If $\lambda$ is a cycle, we first do 1 -surgery on $Q$ to get a punctured torus, and then do 2-surgery with the disk in $S$ determined by $\lambda$, to get a new meridian disk having fewer intersections with the band ( $\mathrm{See}\left[\mathrm{Sc}_{1}, 4.7, \mathrm{Sc}_{2}, 5.6\right.$ ] for details of this construction). 

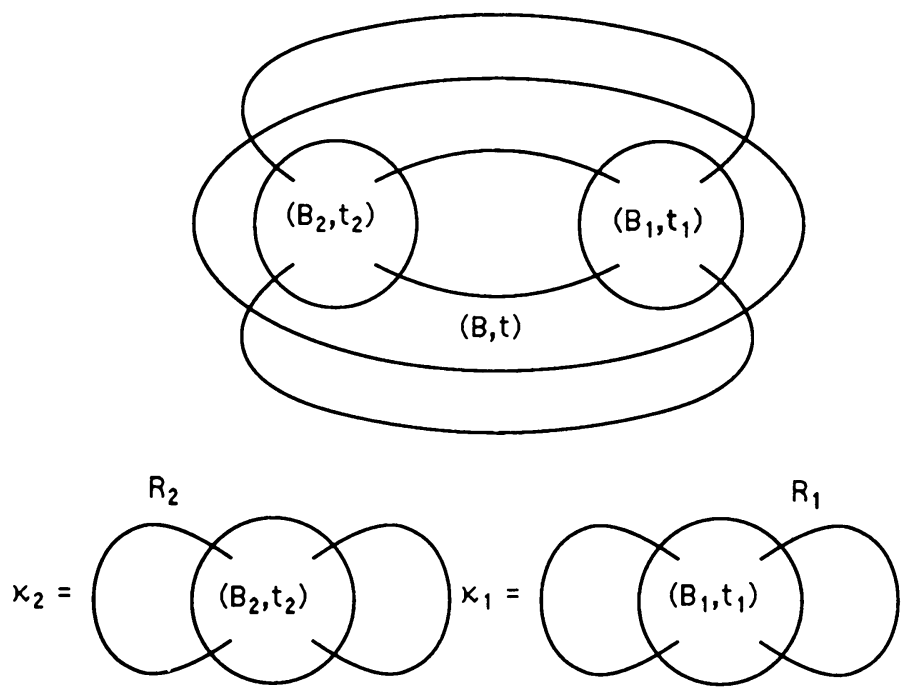

FIGURE 15

This argument implies that the band does not meet $Q$. Doing 2-surgery on $T$ with $Q$, we get a sphere disjoint from the band which intersects $k_{b}$ in two points. This is a decomposing sphere for $k_{b}$, since $k_{b}$ is not a core of $T$, so we have (a) of Theorem 1.

Remark. Actually we are proving a little more: Suppose $k$ is any split link, and $b$ is a band such that $S^{3}-k \cup b(I \times I)$ is irreducible. If the banding $k_{b}$ is composite then the same conclusions as in Theorem 2 happen.

We refer to $[L]$ or $\left[E_{2}\right]$ for definitions and facts about tangles not found here.

Corollary 1. Let $k_{b}, k_{0}, k_{1}$ be as in Theorem 2 . If $k_{b}$ is composite and there is no decomposing sphere disjoint from the band, then $k_{b}$ has two prime summands, and one is a two-bridge knot.

Proof. Let $N$ be a regular neighborhood of the band. It intersects $k_{0} \cup k_{1}$ in two arcs. Its complement can be seen as a locally prime tangle $(B, t)$, i.e. it does not have local summands for no decomposing sphere is disjoint from the band. Then $k_{b}$ and $k_{0} \cup k_{1}$ can be seen as links obtained by summing a rational tangle and $(B, t)$. By Theorem 2 there is a decomposing sphere $P$ intersecting the band in one arc; then $P \cap N$ is a single disk, and $P \cap B$ is disk properly embedded in $(B, t)$ intersecting the strings of $(B, t)$ in two points. This shows that $(B, t)$ is a partial sum of two tangles, say $\left(B_{1}, t_{1}\right)$ and $\left(B_{2}, t_{2}\right)$ (see Figure $15)$. Note that $k_{0} \cup k_{1}$ can be expressed as a tangle sum of $\left(B_{1}, t_{1}\right)$ and $\left(B_{2}, t_{2}\right)$. If both tangles are prime, Theorem 1 in [L] implies that $k_{0} \cup k_{1}$ is a prime link which is impossible, so one of them, say $\left(B_{1}, t_{1}\right)$ is a rational tangle. Let $(N, s)$ be the rational tangle obtained by the intersection of $k_{b}$ and $N . k_{b}$ can be expressed as $k_{b}=(B, t)+(N, s)=\kappa_{1} \# \kappa_{2}$, where $\kappa_{1}=\left(B_{1}, t_{1}\right)+R_{1}$, and $\kappa_{2}=\left(B_{2}, t_{2}\right)+R_{2}$, where $R_{1}, R_{2}$ are rational tangles (see Figure 15). $\kappa_{1}$ is a two-bridge knot because it is the sum of two rational tangles. If $\left(B_{2}, t_{2}\right)$ is also a rational tangle then $\kappa_{2}$ is a two-bridge knot and then it is prime. If $\left(B_{2}, t_{2}\right)$ is a prime tangle, then note that $\left(B_{2}, t_{2}\right)+\left(B_{1}, t_{1}\right)=k_{0} \cup k_{1}$ is a split link and $d\left(\left(B_{1}, t_{1}\right) ; R_{2}\right) \geq 2$ (i.e. the boundaries of the central disks of $\left(B_{1}, t_{1}\right)$ and 
$R_{2}$, which lie in $\partial B_{2}$, meet in at least four points, see [ $\left.\left.\mathrm{E}_{2}\right]\right)$, for otherwise $\kappa_{1}$ would be a trivial knot. Then Theorem 2 in $\left[\mathrm{E}_{2}\right]$ implies that $\kappa_{2}=\left(B_{2}, t_{2}\right)+R_{2}$ is a prime knot.

It is proved in [BE] that if $k_{b}$ is a composite knot and $k_{0}$ and $k_{1}$ are trivial, then one of the summands of $k_{b}$ is a two-bridge knot. (In fact, we do not need the fact that $k_{0}$ and $k_{1}$ are trivial knots, but only that no decomposing sphere is disjoint from the band.)

\section{BANDINGS OF TRIVIAL KNOTS WHICH YIELD COMPOSITE LINKS}

Theorem 3. Suppose $k$ is the trivial knot and $k_{b}$ is a banding of $k$. If $k_{b}$ is a composite knot or link, then there is a decomposing sphere which crosses the band in one arc parallel to $b(\{1 / 2\} \times I)$.

7.1 Proof. Apply Theorem 1, as stated in the introduction. (a) cannot happen because this would imply that $k$ has a nontrivial summand. (c) does not happen for there is no essential torus in the exterior of $k$. So either (b) or (d) happens. If (b) holds we are finished, so suppose we have (d).

\subsection{So assume that:}

There is a disk $Q^{\prime}$ with $\partial Q^{\prime}=k$, so that it has a minimal number of intersections with the band among all such disks, and such that the band intersects it always in the same direction.

Remark. Let $(M, \gamma, \beta)$ as in 1.2. Note that the sutured manifold decomposition $(M, \gamma, \beta) \stackrel{Q^{\prime}}{\longrightarrow}\left(M_{1}, \gamma_{1}, \beta_{1}\right)$ is $\beta$-taut, $\partial M_{1}$ is a sphere with only one suture, and then $\left(M_{1}, \gamma_{1}\right)$ is $\varnothing$-taut; i.e. the sutured manifold hierarchy as constructed in 1.4 consist of only one step.

7.3. Let $P^{\prime}$ be a decomposing sphere for $k_{b}$. In what follows we want to prove that either the band meets $P^{\prime}$ in one arc, or that 7.2 is impossible. Let $N$ be a regular neighborhood of $k \cup b(I \times I)$. The last is homeomorphic to a 3-ball with two 1-handles. By general position $P^{\prime}$ can be isotoped so that $P^{\prime} \cap b(I \times I)=b\left(\left\{a_{i}\right\} \times I\right)$ for some finite $\left\{a_{i}\right\} \subset \operatorname{int}(I)$. Similarly, by general position, $Q^{\prime}$ can be isotoped so that $Q^{\prime} \cap b(I \times I)=b(I \times \partial I) \cup b\left(I \times\left\{b_{s}\right\}\right)$, where $\left\{b_{s}\right\}$ is some finite subset of $I$. Suppose $P^{\prime}$ has a minimal number of intersections arcs with the band. Let $P=P^{\prime}-\operatorname{int}(N), Q=Q^{\prime}-\operatorname{int}(N)$; $P, Q$ are connected planar surfaces in the complement of $N$. $\partial P$ consist of $n$ curves $a_{1}, \ldots, a_{n}$, plus two curves $a_{+}, a_{-}$, which are meridians of the 1handles, as in Figure 16. $\partial Q$ consist of $m$ curves $b_{1}, \ldots, b_{m}$, plus one curve $b_{0}$ which goes once over the 1-handles; we have a situation as in Figure 16. See [ $\mathrm{Sc}_{1}, \mathrm{Sc}_{2}$ ] for more details over $N$ and the positions of the boundary curves of $P$ and $Q$.

Give an orientation to $P$ and $Q$. This induces an orientation in their boundaries. Two curves $a_{i}$ and $a_{j}$ are parallel if with the induced orientation they are parallel in $\partial N$, the same for the $b_{i}$ 's. By 7.2 we may assume that all the $b_{i}$ 's, $1 \leq i \leq m$, are parallel. $b_{0}$ also has an induced orientation. $a_{i}$ and $b_{j}$ meet in two points, one in the front of $N$ and one in the back. Label the point with $i$ in $Q$, and with $j$ in $P$, if this point is in the front of $N$, and label it with $i^{*}$ in $Q$ and $j^{*}$ in $P$ if it is in the back of $N$, for $1 \leq i \leq n, 1 \leq j \leq m$. Label the point of intersection between $b_{0}$ and $a_{i}$ with $i$ in $Q$ and with 0 


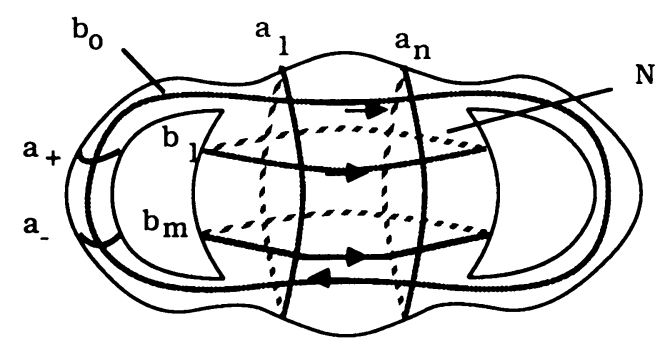

(a)

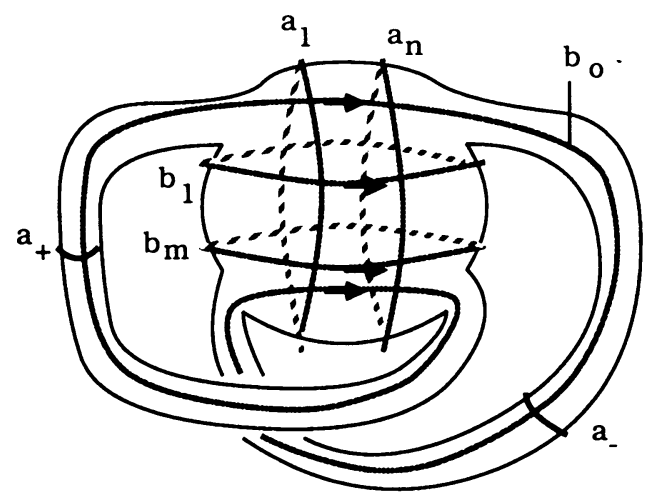

(b)

Figure 16

in $P$ if it lies in the top of $N$, and label it with $i$ in $Q$ and $m+1$ in $P$ if it lies in the bottom of $N$. Denote the points of intersection between $b_{0}$ and $a_{+}, a_{-}$with,+- in both $P$ and $Q$. See Figure 17. Consider the graphs of intersection between $P$ and $Q$ like in $\left[\mathrm{Sc}_{1}\right]$, denote them by $\Gamma_{P}, \Gamma_{Q}$. Define loop, good loop, bad loop, circuit, cycle, $i$-path, Scharlemann cycle, level edge, etc., as in $\left[\mathrm{Sc}_{1}\right]$ or as in $2.2,4.2$. Define the interior of a circuit or path going from $b_{0}$ to $b_{0}$ in $\Gamma_{Q}$ as the component of its complement which does not contain the point $x$ on the east of $b_{0}$ as in the Figure 17.

7.4. We have two cases, that is, $k_{b}$ is either a knot or a link of two components.

Case 1. $k_{b}$ is a link.

In this case $N$ appears as in Figure 16(a). $a_{+}, a_{-}$lie in the same 1-handle, suppose they lie in the west side of $N$, as in Figure 16(a). The top (bottom) part of $b_{0}$ with the induced orientation is either parallel to the front part or to the back part of the $b_{i}$ 's. Suppose with no loss of generality that the top part of $b_{0}$ is parallel to the front part of the $b_{i}$ 's and the bottom part of $b_{0}$ is parallel to the back part of the $b_{i}$ 's. Relabel a point of intersection between $b_{0}$ and $a_{i}$ with $i$ in $Q$ and with 0 in $P$ if this point lies in the top of $N$, and label it with $i^{*}$ in $Q$ and with $(m+1)^{*}$ in $P$ if it lies in the bottom of $N$. It is not difficult to see that $\Gamma_{Q}$ appears as in Figure 18.

7.5. Here we have the same parity rule as in 2.1. Note that in $\Gamma_{Q}$ there is no edge with labels $i-i\left(i^{*}-i^{*}\right)$, i.e. a level edge has to be labeled $i-i^{*}$. Note that the top part of $b_{0}$ has labels $1,2, \ldots, n$, and the bottom has labels $1^{*}, 2^{*}, \ldots, n^{*}$. This implies that a loop in $\Gamma_{P}$ has ends labeled $i-j^{*}$. 

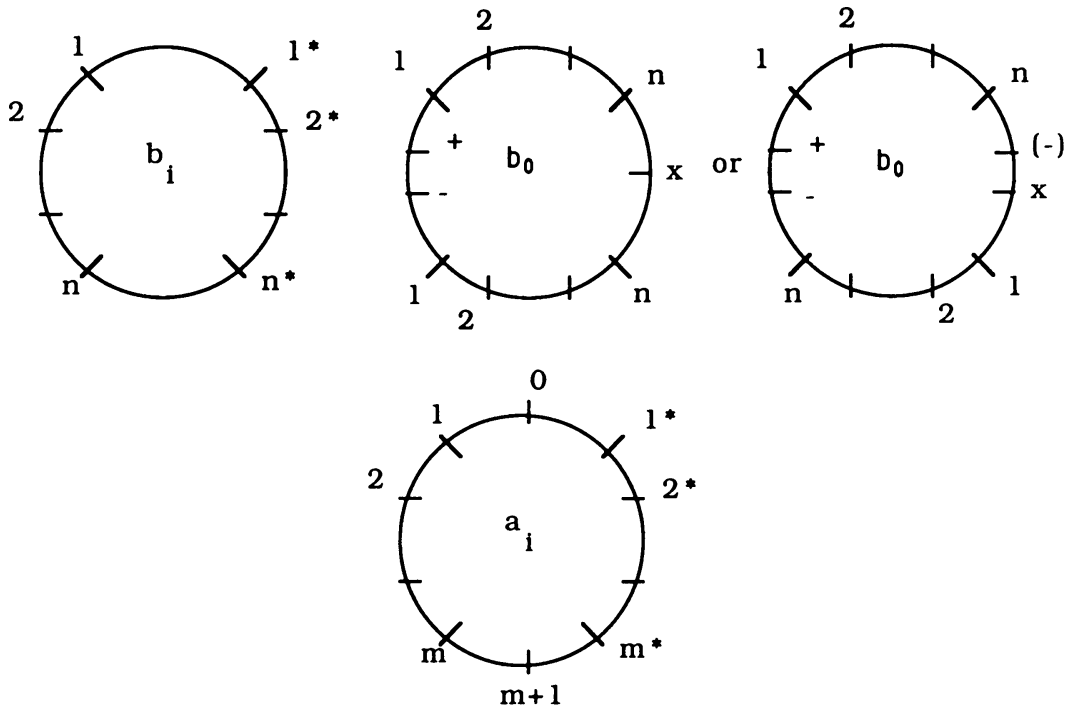

FIGURE 17

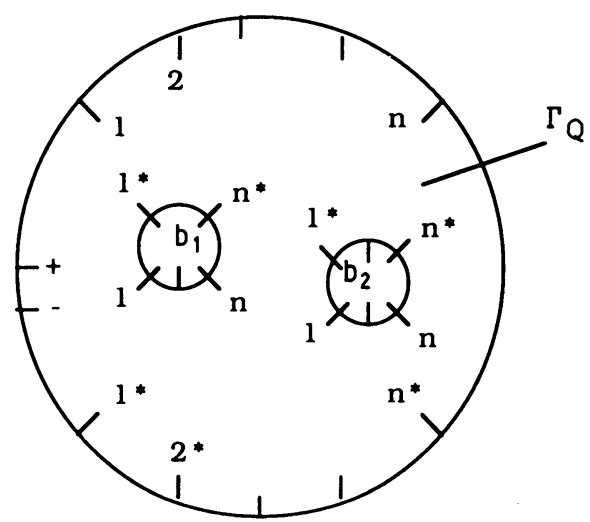

FIGURE 18

7.6 Lemma. A loop (good loop) in $\Gamma_{Q}\left(\Gamma_{P}\right)$ has interior vertices.

Proof. It is as in $\left[\mathrm{Sc}_{2}, 5.1\right]$ or $\left[\mathrm{E}_{1}, 3.2\right]$.

7.7 Lemma. There is no i-cycle in $\Gamma_{Q}$. So there is no Scharlemann cycle in $\Gamma_{Q}$. In particular there is no loop in $\Gamma_{Q}$.

Proof. It is the same as in 2.3, 2.4.

The following lemmas look like 2.7, 4.6 and 4.7.

7.8 Lemma. There is a 1-path (or a $1^{*}$-path) which starts at $b_{0}$ and finishes at + or at - .

Proof. Take a 1-path $\gamma$ starting at $b_{0}$. Because there is no 1-cycle, $\gamma$ either finishes at + or - , or in the other side of $b_{0}$. If $\gamma$ finishes at the bottom part of $b_{0}$, take a $1^{*}$-path $\gamma^{\prime}$ starting at $b_{0}, \gamma^{\prime}$ is in the interior of $\gamma . \gamma^{\prime}$ either finishes at,+- or at $b_{0}$. If $\gamma^{\prime}$ finishes at $b_{0}$ then $\gamma$ and $\gamma^{\prime}$ are just the 
same path, and they have all their edges labeled $1-1^{*}$. In this case if there are vertices in the interior of $\gamma$, there will be an $i\left(i^{*}\right)$-cycle, for some $i$, unless $n=1$, and so we will be done; if there is no vertex in the interior of $\gamma$, then there is a loop joining + and - , which contradicts 7.6. So either $\gamma$ or $\gamma^{\prime}$ finishes at + or - .

Suppose without loss of generality that there is a 1-path $\gamma$ which starts at $b_{0}$ and finishes at + . Note that this path cannot be just an edge joining $b_{0}$ and + .

7.9 Lemma. All the edges adjacent to the top part of $b_{0}$ are level, i.e., for each $i, 1 \leq i \leq n$, there is an edge with label $i$ at $b_{0}$ and label $i^{*}$ at some other vertex.

Proof. Take a $1^{*}$-path $\gamma^{\prime}$ staring at $b_{0}$. It finishes at - or at the other side of $b_{0}$. Suppose first that it finishes at the other side of $b_{0}$. Take now an $i^{*}$-path starting at $b_{0}$ for each $i>1$. As in the proof of Lemma 2.7 each one of these paths finishes at $b_{0}$ with a level edge $i^{*}-i$, for $i \geq 1$. Suppose now that $\gamma^{\prime}$ finishes at - . So - is not in the interior of $\gamma$; this implies that all the edges of $\gamma$ are level and that there is no vertex in its interior, for otherwise there will be a $1^{*}$-cycle in its interior. In particular the edge adjacent to $b_{0}$ with label 1 has label $1^{*}$ in its other end. Take now an $i^{*}$-path starting at $b_{0}$ for each $i>1$, these paths finish at the other side of $b_{0}$, and as before each of these paths finishes with a level edge $i-i^{*}$.

7.10 Lemma. Each vertex in $\Gamma_{P}$ has $m$ bad loops, its labels $m$ and $1^{*}$ are the only ones which are not adjacent to loops.

Proof. 7.9 implies that each vertex in $\Gamma_{P}$ has a loop, and then all the loops are bad, for if there was a good loop there will be one with no interior vertex, contradicting 7.6. All the loops at the same vertex are concentric. There is an edge $\eta$ joining $a_{1}$ and $a_{+}, \eta$ has label $i$ at $a_{1}$, say, $i \neq 0, m+1$. Then because there is a loop in $a_{1}$, there must be a loop with labels $i-1, i+1$; this contradicts the parity rule unless $i=m$. So $\eta$ has label $m$ at $a_{1}$, and there is a loop with labels $m-1,(m+1)^{*}$; by 7.9 there is a loop in $a_{1}$ with labels $0, j^{*}$. The only way that this is possible is that $j^{*}=2^{*}$, and so $1^{*}$ and $m$ are the only labels at $a_{1}$ not adjacent to some loop; then there are $m$ loops at $a_{1}$. Let $\eta_{1}$ be the edge adjacent to $a_{1}$ at the label $1^{*}$. If $\eta_{1}$ meets $a_{-}$ then $n=1$, for otherwise there would be good loops, so suppose $\eta_{1}$ meets a vertex $a_{j}$. Repeating for $a_{j}$ the argument made for $a_{1}$, we get that $\eta_{1}$ has label $1^{*}$ or $m$ at $a_{j}$; the first is not possible for it implies that there is a loop in $\Gamma_{Q}$. Then we conclude that $\eta_{1}$ has label $m$ at $a_{j}$, and that all the labels at $a_{j}$ are adjacent to loops except $1^{*}$. Repeating the same argument for the other vertices completes the proof.

7.11 Conclusion of Case 1. 7.10 shows that all the edges in $\Gamma_{Q}$ with one end labeled 1, have their other end labeled with $1^{*}$, except at $b_{m}$, which has an edge adjacent at its label 1 which meets + . Similarly, all the edges with one end labeled $1^{*}$ have the other end labeled 1 except the edge adjacent to $b_{1}$, which has its other end at $b_{m}$ labeled $j$. This implies that the 1-path $\gamma$ has all its edges with labels $1-1^{*}$, except the last one which joins $b_{m}$ and + . Take a $1^{*}$ path $\gamma^{\prime}$ starting at $b_{0} . \gamma^{\prime}$ has to reach $b_{1}$, for otherwise it could be continued indefinitely. So $\gamma^{\prime}$ reach $b_{1}$ and the next vertex it meets is $b_{m} .7 .10$ implies 


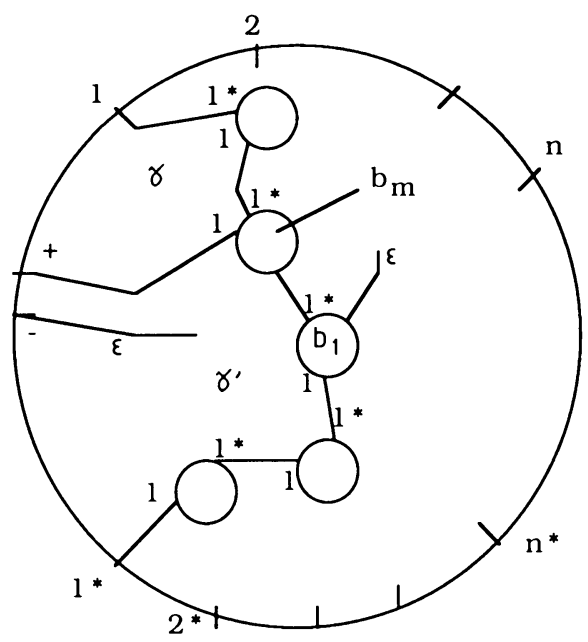

FIGURE 19

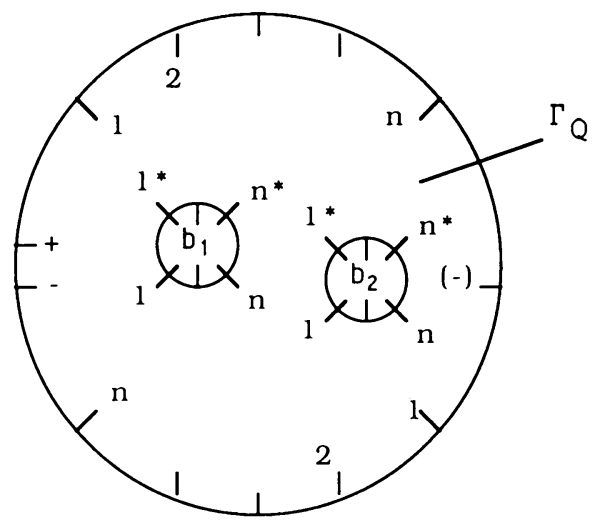

FIGURE 20

that there is an edge $\varepsilon$ joining $b_{1}$ and -, with label $i^{*}$ in $b_{1}$, for some $i$, but this is impossible, as can be seen from Figure 19. This contradiction implies that if $k_{b}$ is a link and 7.2 happens then $n=1$, that is, the band meets the decomposing sphere $P^{\prime}$ in one arc.

7.12 Case 2. $k_{b}$ is a knot. In this case we have a situation as in Figure 16(b). The top and bottom part of $b_{0}$ with the induced orientation are both parallel either to the front part or to the back part of the $b_{i}$ 's. Suppose without loss of generality that they are parallel to the front part of the $b_{i}$ 's. Relabel the points of intersection between $b_{0}$ and $a_{i}$ with $i$ in $\Gamma_{Q}$ and with 0 or $m+1$ in $\Gamma_{P}$, depending if such point is in the top or bottom of $N$. It is not difficult to see that $\Gamma_{Q}$ appears as in Figure 20. It can be seen without difficulty that 7.5, 7.6, 7.7 apply here. Note that $b_{0}$ has only labels $1,2, \ldots, n$, i.e. it has no labels $1^{*}, \ldots, n^{*}$.

7.13 Lemma. For each $i$, there is an $i$-path starting at $b_{0}$ and finishing at + or at - . 
Proof. For each $i$, take an $i$-path starting at the top side of $b_{0}$, say. If the path reaches the other side of $b_{0}$, then its label there is not $i$, and then the path can be continued, and because there is no $i$-cycle in $\Gamma_{Q}$, that path has to reach + . or - .

7.14 Lemma. $\Gamma_{P}$ has at most two vertices.

Proof. Lemma 7.13 implies that $n \leq \#\{+,-\}$.

It can be assumed that there is a 1-path $\gamma_{1}$, starting at the top of part of $b_{0}$ and finishing at + , which is in the west of $b_{0}$; and there is a 2-path $\gamma_{2}$, starting at $b_{0}$ and finishing at - .

7.15 Lemma. $\gamma_{1}$ has only level edges, it has no vertices in its interior, and it has two or more edges.

Proof. If there are vertices in the interior of $\gamma_{1}$ or some edge of $\gamma_{1}$ is not level, then all vertices in the interior of $\gamma_{1}$ (including these vertices in $\gamma_{1}$ which have interior labels) have a label $1^{*}$, and then there is a $1^{*}$-cycle, for a label $1^{*}$ cannot be joined to - . If $\gamma_{1}$ is just an edge joining $b_{0}$ and + , this contradicts the fact that all the loops have interior vertices.

7.16 Conclusion of Case 2. Lemma 7.13 implies that there is an edge $\eta$ in $\Gamma_{P}$ joining $a_{1}$ and $a_{+}$, and 7.15 implies that there is a loop $\lambda$ in $a_{1}$ with labels $0, j^{*}$. If the label of $\eta$ in $a_{1}$ is $i$, then there should be a loop at $a_{1}$ with labels $i-1, i+1$, but this contradicts the parity rule unless $i=m+1 \quad(i=3$ in our case). So $\eta$ has label $m+1$ at $a_{1}$. This implies that there is an edge $\eta$ in $\Gamma_{Q}$ joining the bottom side of $b_{0}$ and + . This is in contradiction with 7.15 , for both $\gamma_{1}$ and $\eta$ reach + , but by construction $\eta$ cannot be part of $\gamma_{1}$. This shows that when $k_{b}$ is a knot 7.2 cannot happen. This completes the proof of Theorem 3.

Corollary 2. Suppose $k$ is the trivial and $k_{b}$ is a composite knot or link. Then $k_{b}$ has two prime summands, and one of them is a two-bridge knot or link.

Proof. It is similar to the proof of Corollary 1. Here we apply $\left[\mathrm{E}_{1}\right]$ (or $\left[\mathrm{E}_{2}\right.$, Theorem 6]) to prove that one of the summands is prime.

S. A. Bleiler has proved in $\left[\mathrm{B}_{2}\right]$, using a purely combinatorial argument, that if a banding $k_{b}$ of a trivial knot $k$ is composite, then $k_{b}$ has a two-bridge summand.

7.17. It is asked in [L] how many ways there are of summing a rational tangle to a given prime tangle to get a nonprime knot or link. The first examples of prime tangles admitting two different summings of a rational tangle which yield a nonprime knot or link were given in $\left[B_{1}\right]$. In [ $E_{2}$, Corollary 1] it is shown that there are at most three such summings of rational tangles, and they are at a distance 1 from each other, i.e. they differ by a half twist. Theorems 2,3 give the following refinement:

Corollary 3. Let $(B, t)$ be a prime tangle. Suppose it can be summed with a rational tangle to get the trivial knot or a split link. Then there is at most one more way of summing a rational tangle to get a nonprime knot or link (in fact it has to be a composite one). If this happens then $(B, t)$ is a partial sum of two tangles, one of them a rational tangle. 
Proof. Suppose that $(B, t)+\left(B_{1}, t_{1}\right)=k_{1}$ is the trivial knot or a split link, for $\left(B_{1}, t_{1}\right)$ a rational tangle. If there is another way of summing a rational tangle $\left(B_{2}, t_{2}\right)$ to $(B, t)$ to get a nonprime knot or link $k_{2}$, then by [ $\mathrm{E}_{2}$, Theorem 3$]$, [Sc ${ }_{1}$ or [BS] $k_{2}$ has to be composite. Now by [E, Theorems 2,6$],\left(B_{1}, t_{1}\right)$ and $\left(B_{2}, t_{2}\right)$ are at distance 1 , then $k_{2}$ is obtained by a banding of $k_{1}$. So by Theorems 2, 3 there is a decomposing sphere $P_{2}$ for $k_{2}$ so that $P_{2} \cap B_{2}$ is a single disk, and $P_{2} \cap B$ is a disk meeting $t$ in two points, so $(B, t)$ is a partial sum of two tangles, and as in the proof of Corollary 1 one of the tangles is a rational one. If for another rational tangle $\left(B_{3}, t_{3}\right)$ we have that $(B, t)+\left(B_{3}, t_{3}\right)=k_{3}$ is another nonprime link, then as before $k_{3}$ is composite, $\left(B_{3}, t_{3}\right)$ is at distance 1 from $\left(B_{1}, t_{1}\right)$, and there is a decomposing sphere $P_{3}$ for $k_{3}$ such that $P_{3} \cap B_{3}$ is a single disk. By [E $\mathrm{E}_{2}$, Theorem 1] $\left(B_{2}, t_{2}\right)$ and $\left(B_{3}, t_{3}\right)$ are also at distance 1 . Now it is not difficult to observe that it is not possible that both decomposing spheres $P_{2}$ and $P_{3}$ intersect $B_{2}$ and $B_{3}$ respectively in one disk. This observation also follows from $\left[E_{2}, 2.6,2.7\right]$.

Remark. In the case when we have a trivial knot and a composite link, the disk which realizes the partial sum of the tangles intersects only one of the strings of $(B, t)$, so this is not a partial sum in the sense of [L].

This corollary shows that if there is a prime tangle which yields three nonprime knots or links by attachments of rational tangles, then these knots and links have to be composite, but it is still unknown if such a tangle exists. A positive solution to the following conjecture would imply that such a tangle does not exist.

Conjecture. Let $k$ be $a$ link and $b$ a band. Suppose that $k$ and $k_{b}$ are both composite. Suppose that there is no decomposing sphere for $k$ or $k_{b}$ disjoint from the band. Then there is a decomposing sphere for $k$ or one for $k_{b}$ which intersects the band in one arc parallel to $b(I \times\{1 / 2\})$ or to $b(\{1 / 2\} \times I)$.

\section{SURGERY ON STRONGLY INVERTIBLE KNOTS}

In this section we apply Theorem 3 to surgery on a knot. We refer to [R] for definitions and facts about Dehn surgery.

It is known that $(p q, 1)$ surgery on a $(p, q)$-cable knot gives a nonprime manifold with a lens space summand (see $[\mathrm{G}]$ ). It is conjectured in [GoS] that those are all the surgeries on a knot which yield a reducible manifold. In other words, the conjecture says that if surgery on a knot in $S^{3}$ yields a reducible 3manifold then the knot is cabled, with the cabling annulus part of the reducing sphere. The conjecture has been proved for several classes of knots, in fact it was recently proved for alternating knots [MT]. See [ $\left.\mathrm{Sc}_{4}\right]$ or $\left[\mathrm{E}_{3}\right]$ for more references in this problem. Here we prove the conjecture for strongly invertible knots.

Definition. A knot $k$ in $S^{3}$ is strongly invertible if there is an orientation preserving involution of $S^{3}$ which carries $k$ to itself, reversing its orientation. Waldhausen [W] shows that such an involution is equivalent to a $\pi$-rotation whose axis meets $k$ in exactly two points.

Theorem 4. Suppose $k$ is a nontrivial strongly invertible knot in $S^{3}$. If some surgery on $k$ yields a reducible manifold then $k$ is cabled, and the surgery is via the slope of the cabling annulus. 
Proof. Let $M(k ; r)$ denote the closed 3-manifold obtained by Dehn surgery with coefficient $r$ on $k$. As $k$ is strongly invertible, $M(k ; r)$ double branch covers the 3-sphere [M]. Furthermore the base space and branch set $B(k ; r)$ of the double branch covering $p: M(k ; r) \rightarrow S^{3}$ decomposes, in an appropriate projection, as the $r$-rational tangle attached to a locally prime tangle $\mathbb{B}=(B, t)$, and so that $\mathbb{B}$ plus the $1 / 0$-rational tangle is the trivial knot $\left[B_{1}\right] . \mathbb{B}$ is in fact a prime tangle if $k$ is nontrivial.

Let $M=M(k ; r)$ be a reducible manifold. Let $\kappa$ denote the knot or link of two components which is the branch set of the respective cover. Suppose first that $M=S^{1} \times S^{2} \# M^{\prime}$. [KT, Corollary 4] says that a link is prime if and only if its double branch cover is irreducible. So $\kappa$ is either split or composite. If $\kappa$ is composite but nonsplit, say $\kappa=\kappa_{1} \# \kappa_{2}$, then its double branch cover is $M=M_{1} \# M_{2}$, where $M_{i}$ is the double branch cover of $\kappa_{i}$, so this would imply that $S^{1} \times S^{2}$ is the double branch cover of a nonsplit link, which contradicts the fact that $S^{1} \times S^{2}$ uniquely double branch covers the unlink [T]. So $\kappa$ is a split link, and $\kappa$ is obtained by summing $\mathbb{B}$ and the 0 -rational tangle. But $\mathbb{B}$ plus the 1/0-rational tangle is the trivial knot, so this says that the unknot can be obtained by a band sum of a split link; [ $\left.\mathrm{Sc}_{1}\right]$ implies that the band and the split link have to be trivial, and then $\mathbb{B}$ is in fact a trivial tangle, implying that $k$ is the trivial knot.

Suppose now that $M$ is reducible but does not contain $S^{1} \times S^{2}$ as a summand. Then [KT, Corollary 4] implies that $\kappa$ is a composite link. $\kappa$ is obtained by summing $\mathbb{B}$ and the $r$-rational tangle, then $\left[E_{1}\right]$ implies that $r$ is an integer. This is equivalent to saying that $\kappa$ is obtained by a banding of the trivial knot. Theorem 3 implies that there is a decomposing sphere $P$ for $\kappa$ so that $P \cap(r$-rational tangle $)$ is a disk disjoint from $\kappa$, and $P \cap B$ is a disk meeting $\kappa$ twice. $A=p^{-1}(P \cap B)$ is an annulus, and each one of its boundary components is a circle of slope $r$ on $\partial \eta(k)$, where $r$ is integer. It is not difficult to see that then $k$ is a cable knot and the slope of the surgery which yields $M$ is that of the annulus.

Theorem 2 and $\left[E_{2}\right]$ can be used to get results about Dehn fillings on manifolds which are double covers of a prime tangle $(B, t)$ branched along $t$; see $\left[\mathrm{E}_{3}\right]$

\section{REFERENCES}

$\left[\mathrm{B}_{1}\right] \quad$ S. A. Bleiler, Prime tangles and composite knots, Knot Theory and Manifolds, Lecture Notes in Math., vol. 1144, Springer-Verlag, Berlin and New York, 1985, pp. 1-13.

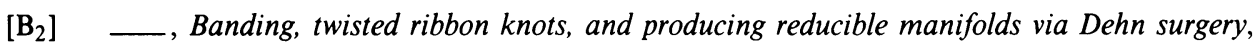
Math. Ann. 286 (1990), 679-696.

[BE] S. A. Bleiler and M. Eudave-Muñoz, Composite ribbon number one knots have two-bridge summands, Trans. Amer. Math. Soc. 321 (1990), 231-244.

[BS] S. A. Bleiler and M. Scharlemann, A projective plane in $R^{4}$ with three critical points is standard. Strongly invertible knots have property $P$, Topology 27 (1988), 519-540.

[CGLS] M. Culler, C. Gordon, J. Luecke, and P. Shalen, Dehn Surgery on knots, Ann. of Math. 125 (1987), 237-300.

[E $\left.E_{1}\right]$ M. Eudave-Muñoz, Cirugia en nudos fuertemente invertibles, An. Inst. Mat. Univ. Nac. Autonoma de Mexico 26 (1986), 41-57.

$\left[\mathrm{E}_{2}\right] \quad \ldots$, Primeness and sums of tangles, Trans. Amer. Math. Soc. 306 (1988), 773-790. 
$\left[\mathrm{E}_{3}\right] \ldots$ Band sums of knots and composite links. The cabling conjecture for strongly invertible knots, Ph.D. thesis, UCSB, 1990.

[GoS] F. Gonzalez-Acuña and H. Short, Knot surgery and primeness, Math. Proc. Cambridge Philos. Soc. 102 (1986), 89-102.

[G] C. Gordon, Dehn surgery and satellite knots, Trans. Amer. Math. Soc. 275 (1983), 687-708.

[H] J. Hempel, 3-manifolds, Ann. of Math. Studies, no. 86, Princeton Univ. Press, Princeton, N.J., 1976.

[KT] P. K. Kim and J. L. Tollefson, Splitting the P. L. involutions of nonprime 3-manifolds, Michigan Math. J. 27 (1980), 259-274.

[L] W. B. R. Lickorish, Prime knots and tangles, Trans. Amer. Math. Soc. 267 (1981), 321-332.

[MT] W. Menasco and M. Thistlethwaite, Surfaces with boundary in alternating knots exteriors, preprint.

[M] J. M. Montesinos, Surgery on links and double branched covers of $S^{3}$, Ann. of Math. Studies, no. 84, Princeton Univ. Press, Princeton, N.J., 1975, pp. 227-260.

[R] D. Rolfsen, Knots and links, Publish or Perish, 1976.

[Sc $\left.\mathrm{Sc}_{1}\right] \quad \mathrm{M}$. Scharlemann, Smooth spheres in $R^{4}$ with four critical points are standard, Invent. Math. 79 (1985), 125-141.

[Sc $\left.\mathrm{Sc}_{2}\right]$ - Unknotting number one knots are prime, Invent. Math. 82 (1985), 37-55.

[ $\left.\mathrm{Sc}_{3}\right] \quad$, Sutured manifolds and generalized Thurston norms, J. Differential Geometry 29 (1989), 557-614.

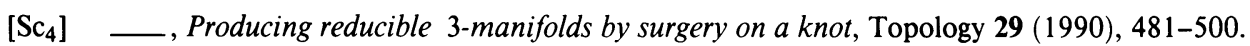

[ST $\mathrm{ST}_{1}$ M. Scharlemann and A. Thompson, Unknotting number, genus, and companion tori, Math. Ann. 280 (1988), 191-205.

[ST $\left.{ }_{2}\right] \ldots$ Link genus and the Conway moves, Comment. Math. Helv. 64 (1989), 527-535.

[T] J. L. Tollefson, Involutions on $S^{1} \times S^{2}$ and other 3-manifolds, Trans. Amer. Math. Soc. 183 (1973), 139-152.

[W] F. Waldhausen, Über Involutionen der 3-sphare, Topology 8 (1969), 81-91.

School of Mathematics, Institute for Advanced Study, Princeton, New Jersey 08540

Current address: Instituto de Matematicas, Universidad Nacional Autonoma de Mexico, 04510 Mexico D.F., Mexico 